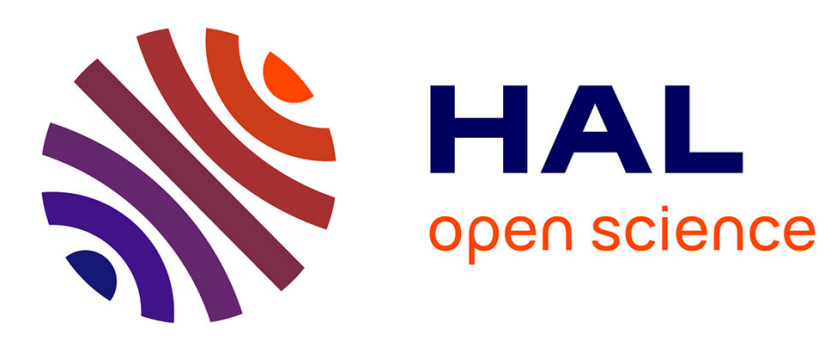

\title{
On the performance of a high-order multiscale DG approach to LES at increasing Reynolds number
}

\author{
Marta de La Llave Plata, Eric Lamballais, Fabio Naddei
}

\section{To cite this version:}

Marta de La Llave Plata, Eric Lamballais, Fabio Naddei. On the performance of a high-order multiscale DG approach to LES at increasing Reynolds number. 2019. hal-02197477v2

\section{HAL Id: hal-02197477 \\ https://hal.science/hal-02197477v2}

Preprint submitted on 6 Sep 2019

HAL is a multi-disciplinary open access archive for the deposit and dissemination of scientific research documents, whether they are published or not. The documents may come from teaching and research institutions in France or abroad, or from public or private research centers.
L'archive ouverte pluridisciplinaire HAL, est destinée au dépôt et à la diffusion de documents scientifiques de niveau recherche, publiés ou non, émanant des établissements d'enseignement et de recherche français ou étrangers, des laboratoires publics ou privés. 


\title{
On the performance of a high-order multiscale DG approach to LES at increasing Reynolds number
}

\author{
Marta de la Llave Plata ${ }^{\mathrm{a}, *}$, Eric Lamballais ${ }^{\mathrm{b}}$, Fabio Naddei ${ }^{\mathrm{a}}$ \\ ${ }^{a}$ ONERA - Université Paris-Saclay, 92320 Châtillon, France \\ ${ }^{b}$ Université de Poitiers, CNRS, ISAE-ENSMA, 86962 Futuroscope Chasseneuil, France
}

\begin{abstract}
The variational multiscale (VMS) approach based on a high-order discontinuous Galerkin (DG) method is used to perform LES of the sub-critical flow past a circular cylinder at Reynolds 3900, 20000 and 140000 . The effect of the numerical flux function on the quality of the LES solutions is also studied in the context of very coarse discretizations of the TGV configuration at $R e=20000$. The potential of using $p$-adaption in combination with DG-VMS is illustrated for the cylinder flow at $R e=140000$ by considering a non-uniform distribution of the polynomial degree based on a recently developed error estimation strategy [59]. The results from these tests demonstrate the robustness of the DG-VMS approach with increasing Reynolds number on a highly curved geometrical configuration.
\end{abstract}

Keywords: Discontinuous Galerkin method, large-eddy simulation, variational multiscale approach, $p$-adaptation, cylinder flow

\section{Introduction}

Thanks to the exponential increase in cheap processing power over the last decade and GPU technology advancing at a fast pace, the large-eddy simulation (LES) technique, which for many years has remained unaffordable at the industrial scale, is becoming within reach of the aerospace industry.

Even though the Reynolds-averaged Navier-Stokes (RANS) approach constitutes today the main simulation tool for a wide range of aeronautical configurations, the inability of RANS models to accurately predict massively separated flows and largescale unsteady phenomena restricts their applicability to steady or mildly unsteady flow problems. In the context of highly unsteady configurations, the large-eddy simulation (LES) technique therefore represents an attractive alternative to RANS.

The derivation of the LES equations is based on the concept of spatial filtering or projection, by which the turbulent field is decomposed into a large-scale component, and a subgrid-scale (SGS) component, representative of the small scales of turbulence.

\footnotetext{
${ }^{*}$ Corresponding author

Email address: marta.de_la_llave_plata@onera.fr (Marta de la Llave Plata)
} 
In LES, the large scales are explicitly computed, whereas the effect of the small scales on the resolved field is modelled. In general, it is expected that the large scales captured by LES will provide an accurate prediction of the kinetic energy, or equivalently, that its SGS component will be small. This is why LES requires highly accurate numerical schemes and considerably higher resolution levels than those typical of RANS simulations, for which only mean flow quantities have to be computed.

In particular, the variational multiscale (VMS) approach to LES [31] deserves some attention, thanks to its ability to control the excessive dissipation introduced at the largescale level, which is one of the main drawbacks of most commonly used SGS models. The main idea behind the VMS method is thus to restrict the effect of the SGS model to a small range of resolved scales, called the small resolved scales, by means of high-pass filtering or projection. A fundamental assumption of this approach is therefore that the effect of the unresolved scales on the large resolved scales can be neglected. This feature is particularly interesting for the simulation of transitional flows [21], as those that will be considered in this paper.

The VMS approach was originally proposed by Hughes et al. [31] as a framework for constructing stable finite-element methods (FEM) for approximating multiscale physical phenomena. The first formulation of VMS in the context of LES was published a little later [32]. Concurrently, Collis proposed in [17] a reformulation of the formalism introduced in [32], based on a three-scale decomposition of the solution, and clarified the role the SGS model in the VMS formulation. The first application of VMS to LES of incompressible turbulence was based on spectral methods and was reported in $[32,33$, $34,65]$. In these early studies, the separation of scales is straightforwardly performed in spectral space by employing a sharp cutoff filter. An extension of VMS to unstructured grids was soon proposed by Jansen and Tejada-Martínez [39], in the context of a continuous Galerkin (CG) FEM based on hierarchical basis functions. Scale-separation was here achieved via splitting of the hierarchical bases into low-order polynomials, associated with the large resolved scales, and high-order polynomials, associated with the small resolved scales. This methodology was assessed on a homogeneous isotropic turbulence configuration, considering hexahedral and tetrahedral meshes. Still in the context of CG-FEM, John and Kaya [40] put forward a VMS formalism in which the separation of scales is performed in the reference FE space, very similar in spirit to the procedure proposed in [39]. In [40], the authors apply the proposed method to the simulation of the three-dimensional mixing layer problem and the flow past a square cylinder. Wasberg et al. [75] have also proposed a VMS approach for the spectral element method (SEM) based on tensor products of Lagrangian interpolants of Legendre polynomials. The scale decomposition is then carried out using the modal representation of the solution via appropriate operators. In [75], the method was assessed on the turbulent channel flow at $R e_{\tau}=180,550$ and 950. A VMS approach in the framework of the finite-volume method (FVM) has also been developed by Gravemeier [27]. This approach relies on the use of multigrid operators to carry out the splitting of the resolved scales into large and small, and was effectively applied to perform LES of the incompressible flow in a diffuser. Since then, such methods have been successfully applied to the simulation of incompressible turbulence by means of various numerical methods and in combination with different SGS models (see [2] and [69] for an exhaustive review). 
A compressible formulation of the VMS approach on unstructured meshes was independently proposed by Koobus and Farhat [42] and by Levasseur et al. [43]. The methodology proposed in [42] relies on a grid-agglomeration procedure in the context of a hybrid FE/FV method. A Germano-like dynamic version of this approach which dynamically adapts the model constant was later proposed in [23]. This methodology has been applied to study the aerodynamics of bluff bodies in a number of works [66, 56, 38]. On the other hand, the VMS method developed in [43] employs a stabilised FEM based on a Galerkin/least-squares (GLS) approach to discretize the compressible $\mathrm{N}-\mathrm{S}$ equations in entropy variables. Scale separation in VMS is achieved by the use of a second-order Taylor expansion of the Gaussian filter. The proposed VMS formalism was applied to the simulation of freely decaying isotropic turbulence in the limit of infinite Reynolds numbers. More recently, Yser et al. [78] and Yser and Bailly [79] have also used the GLS-FEM and third-order elements to perform hybrid RANSVMS simulations of a three-element high-lift airfoil using VMS. In contrast to the methodology reported in [43], the scale decomposition is now carried out by extracting the low-order component of the solution, which is expressed in terms of polynomial interpolating functions. As mentioned by the authors in [79], this approach considerably reduces the computational overhead associated with the scale separation step in VMS. In the context of the SEM, Munts et al. [57] have proposed a compressible formulation of the VMS method based on the use of high-order hierarchical modal basis functions. In this modal setting, the decomposition of the resolved scales becomes straightforward. In [57] the authors performed up to fourth-order LES simulations of a turbulent channel flow at $R e_{\tau}=180$. This same methodology was later successfully employed in the research reported in [35].

The potential of the VMS approach in the context of high-order discontinuous methods is, however, far from being fully exploited. Indeed, high-order discontinuous compact methods (Discontinuous Galerkin, Spectral Differences, Flux Reconstruction, etc.) provide increased flexibility with respect to classical low-order FEM and FVM. In particular, the choice of the local scale-separation parameter, which sets the limit between the large and the small resolved scales, can be conveniently adapted to the local resolution requirements of the flow, as has been recently demonstrated in a-priori, as well as in dynamic tests of the 3D Taylor-Green vortex (TGV) configuration at $R e=5000$ [58].

Discontinuous Galerkin (DG) methods are high-order variational methods that relax the condition of continuity of the polynomial approximation by penalising discontinuities through a choice of numerical flux shared across element boundaries. This structure has many practical benefits, in particular, the excellent scalability they offer for unsteady problems with explicit time stepping [16].

The variational framework provided by the high-order modal DG approach allows for a straightforward separation of scales using the polynomial basis functions, making this type of discretization a very appealing tool for LES using multiscale methods. The first use of VMS in the framework of DG methods has been reported by Collis [18]. The modal DG method in combination with the VMS approach based on the Smagorinsky model has been successfully applied to the TGV configuration at $R e=3000$ in [13, $15,7]$ and to the circular cylinder configuration at $R e=3900$ in [7]. In [1], Abbà et al. have developed an anisotropic dynamic VMS model in the context of a high-order DG 
method for compressible LES. To this end, the authors exploit the hierarchical nature of the FE basis to define the grid and test filters via projection operators. The proposed dynamic approach is applied in [1] to the plane channel configuration at $M=0.2,0.7$ and 1.5 and to the 2D periodic hill test case at $R_{b}=3900$ and $M=0.2$. In [4], Bando et al. have performed computations of compressible homogeneous isotropic turbulence, showing the good performance of the DG-VMS approach with respect to an implicit LES approach based on an entropy-bounded DG scheme [50]. Based on the aforementioned works, Navah et al. [62] have recently derived a general formulation that extends the VMS method for compressible LES to the family of compact nodal methods represented by the high-order flux reconstruction scheme [36, 37]. Finally, the spectral properties of the DG-VMS approach have been studied in detail by Naddei et al. [58]. The outcome of this study opens new perspectives on the use of VMS in the context of high-order methods.

In this work, the scale-resolving capabilities of a high-order modal DG method [14, 20] are illustrated on a number of turbulent flow configurations. In particular, we evaluate the performance of the DG-VMS technique on the TGV configuration at $R e=20000$ and for the flow past a circular cylinder at $R e=3900,20000$, and 140000 . The latter test case constitutes a more complex configuration, involving real boundary conditions and flow separation. The no-model as well as the standard LES approaches are also considered and compared to DG-VMS for $R e=3900$. The results from the DG simulations are assessed by detailed comparisons against reference data from CFD and experiment. The effect of the numerical flux function on the different terms involved in the kinetic energy balance equation is also studied in the case of the TGV configuration at $R e=20000$. Finally, the potential of using static $p$-adaptation in combination with DG-VMS is illustrated for the cylinder flow configuration at $R e=140000$ by considering a non-uniform distribution of the polynomial degree based on a recently developed error estimator [59]. We will see in Sec. 6.5 that for this more challenging configuration the use of $p$-adaptation allows us to increase the accuracy of the computed solution, while keeping the number of degrees of freedom (DOFs) at a reasonable level. The use of a static $p$-adaptive strategy is in this case justified by the statistically steady character of this flow. In practice, $p$-adaptation is performed in the inhomogeneous streamwise and cross-stream directions only, whereas in the homogeneous spanwise direction $p$ is kept constant.

This paper is organised as follows. After introducing the governing equations in Sec. 2, the standard LES and VMS formulations based on a modal DG method are presented in Secs. 3 and 4. In the framework of the TGV configuration, the focus of Sec. 5 is to study the effect of the flux functions used in the DG method on the accuracy of the solutions. Section 6 reports the results from the DG-LES simulations of the cylinder flow configuration for the three Reynolds numbers considered. Finally, Sec. 7 lays out the main conclusions drawn from this research. 


\section{Governing equations}

Let $\Omega \subset \mathbb{R}^{3}$ be a bounded domain. The compressible Navier-Stokes (N-S) equations, with appropriate boundary conditions in $\partial \Omega$, read

$$
\frac{\partial \mathbf{u}}{\partial t}+\boldsymbol{\nabla} \cdot\left(\mathcal{F}_{c}(\mathbf{u})-\mathcal{F}_{v}(\mathbf{u}, \boldsymbol{\nabla u})\right)=0, \text { in } \Omega
$$

where $\mathbf{u}=(\rho, \rho \mathbf{v}, \rho E)^{T}$ is the vector of conservative variables. The vectors $\mathcal{F}_{c}$, and $\mathcal{F}_{v}$ are the convective and viscous fluxes, respectively,

$$
\mathcal{F}_{c}=(\rho, \rho \mathbf{v} \otimes \mathbf{v}+p \mathbf{I},(\rho E+p) \mathbf{v})^{T}, \mathcal{F}_{v}=(0, \boldsymbol{\tau}, \boldsymbol{\tau} \cdot \mathbf{v}-\mathbf{q})^{T}
$$

In (2), $\boldsymbol{\tau}$ represents the shear-stress tensor, given by

$$
\boldsymbol{\tau}=\mu\left(2 \mathbf{S}-\frac{2}{3}(\nabla \cdot \mathbf{v}) \mathbf{I}\right) \quad \text { with } \quad \mathbf{S}=\frac{1}{2}\left(\nabla \mathbf{v}+\nabla \mathbf{v}^{T}\right)
$$

$\mu$ is the dynamic viscosity and $\mathbf{S}$ the rate-of-strain tensor. The heat-flux vector is written as $\mathbf{q}=-k \nabla T$, with $T$ the temperature and $k$ the thermal diffusivity.

\section{The DG-LES formulation}

Let $\Omega_{h}$ be a shape-regular partition of the domain $\Omega$, into $N$ non-overlapping and non-empty cells $K$ of characteristic size $h$. We also define the sets $\mathcal{E}_{i}$ and $\mathcal{E}_{b}$ of interior and boundary faces in $\Omega_{h}$, respectively, such that $\mathcal{E}_{h}=\mathcal{E}_{i} \cup \mathcal{E}_{b}$. Let $\mathcal{V}_{h}^{p}=\left\{\phi \in L^{2}\left(\Omega_{h}\right):\left.\phi\right|_{K} \in \mathcal{P}^{p}(K), \forall K \in \Omega_{h}\right\}$ be the functional space of piecewise polynomials of degree at most $p$, and $\left(\phi_{K}^{1}, \ldots, \phi_{K}^{N_{p}}\right) \in \mathcal{P}^{p}(K)$ a hierarchical and orthonormal modal basis of $\mathcal{V}_{h}^{p}$, of dimension $N_{p}$, confined to $K$ [6]. The solution in each element is thus expressed as a linear expansion of basis functions, the coefficients of which constitute the degrees of freedom (DOFs) of the problem at hand. The LES technique used in this research is based on the projection of the N-S equations onto the functional space $\mathcal{V}_{h}^{p}$. This projection operation implicitly defines a partitioning of the solution such that a turbulent field $\mathbf{u}$ is decomposed into its resolved, $\mathbf{u}_{h}$, and unresolved components, $\mathbf{u}-\mathbf{u}_{h}$. The variational form of the LES equations thus reads: find $\mathbf{u}_{h}$ in $\mathcal{V}_{h}^{p}$ such that $\forall \phi_{h} \in \mathcal{V}_{h}^{p}$ we have

$$
\frac{\partial}{\partial t} \int_{\Omega_{h}} \phi_{h} \mathbf{u}_{h} d V+\mathcal{L}_{c}\left(\mathbf{u}_{h}, \phi_{h}\right)+\mathcal{L}_{v}\left(\mathbf{u}_{h}, \phi_{h}\right)=\mathcal{L}_{s g s}\left(\mathbf{u}, \mathbf{u}_{h}, \phi_{h}\right)
$$

where we have used the definition of the $L^{2}$-projection, i.e. $\int_{\Omega_{h}}\left(\mathbf{u}-\mathbf{u}_{h}\right) \phi_{h}=0, \forall \phi_{h} \in$ $\mathcal{V}_{h}^{p}$. In Eqn. (4) $\mathcal{L}_{c}$ and $\mathcal{L}_{v}$ represent the weak form of the convective and viscous terms, respectively. The term $\mathcal{L}_{s g s}$ on the right-hand-side of Eqn. (4), is the variational form of the SGS residual representing the effect of the unresolved scales $\mathbf{u}-\mathbf{u}_{h}$ on the resolved field $\mathbf{u}_{h}$.

We now introduce the following notation: for a given interface in $\mathcal{E}_{i}$ we define the average operator $\left\{\{\mathbf{u}\}=\left(\mathbf{u}^{+}+\mathbf{u}^{-}\right) / 2\right.$ and the jump operator $\llbracket \mathbf{u} \rrbracket=\mathbf{u}^{+} \otimes \mathbf{n}-\mathbf{u}^{-} \otimes \mathbf{n}$, 
where $\mathbf{u}^{+}$and $\mathbf{u}^{-}$are the traces of the variable vector $\mathbf{u}$ at the interface between elements $K^{+}$and $K^{-}$, and $\mathbf{n}$ denotes the unit outward normal vector to an element $K^{+}$. The DG discretization of the convective terms reads

$$
\begin{aligned}
\mathcal{L}_{c}\left(\mathbf{u}_{h}, \phi_{h}\right) \cong & -\int_{\Omega_{h}} \mathcal{F}_{c}\left(\mathbf{u}_{h}\right) \cdot \nabla_{h} \phi_{h} d V \\
& +\int_{\mathcal{E}_{i}} \llbracket \phi_{h} \rrbracket \mathbf{h}_{c}\left(\mathbf{u}_{h}^{+}, \mathbf{u}_{h}^{-}, \mathbf{n}\right) d S+\int_{\mathcal{E}_{b}} \phi_{h}^{+} \mathcal{F}_{c}\left(\mathbf{u}_{b}\left(\mathbf{u}_{h}^{+}, \mathbf{n}\right)\right) \cdot \mathbf{n} d S
\end{aligned}
$$

where the boundary values $\mathbf{u}_{b}=\mathbf{u}_{b}\left(\mathbf{u}_{h}^{+}, \mathbf{n}\right)$ are computed so that the boundary conditions are satisfied on $\mathcal{E}_{b}$. In this paper, a modified version of the local Lax-Friedrichs (LLF) flux [71] and the Roe scheme [70] has been employed, which reads

$$
\mathbf{h}_{c}\left(\mathbf{u}_{h}^{+}, \mathbf{u}_{h}^{-}, \mathbf{n}\right)=\frac{1}{2}\left(\mathcal{F}_{c}\left(\mathbf{u}_{h}^{+}\right) \cdot \mathbf{n}+\mathcal{F}_{c}\left(\mathbf{u}_{h}^{-}\right) \cdot \mathbf{n}+\alpha \mathcal{D}\left(\mathbf{u}_{h}^{+}, \mathbf{u}_{h}^{-}, \mathbf{n}\right)\right)
$$

where $\mathcal{D}\left(\mathbf{u}_{h}^{+}, \mathbf{u}_{h}^{-}, \mathbf{n}\right)$ is the upwinding dissipation function associated with the selected numerical flux. This is scaled by a coefficient $\alpha \in[0,1]$ to calibrate the amount of numerical dissipation introduced. In the limiting case of $\alpha=0$ we have a (non dissipative) central scheme, while for $\alpha=1$ we recover the standard version of the flux functions $[71,70]$.

The discretization of the viscous terms is performed using the symmetric interior penalty (SIP) method originally proposed by Arnold et al. [3],

$$
\begin{aligned}
& \mathcal{L}_{v}\left(\mathbf{u}_{h}, \phi_{h}\right) \cong \int_{\Omega_{h}} \mathcal{F}_{v}\left(\mathbf{u}_{h}, \nabla_{h} \mathbf{u}_{h}\right) \cdot \nabla_{h} \phi_{h} d V \\
& -\int_{\mathcal{E}_{i}} \llbracket \phi_{h} \rrbracket\left\{\left\{\mathcal{F}_{v}\left(\mathbf{u}_{h}, \nabla_{h} \mathbf{u}_{h}\right)\right\}\right] \cdot \mathbf{n} d S-\int_{\mathcal{E}_{b}} \phi_{h}^{+} \mathcal{F}_{v}\left(\mathbf{u}_{b}, \nabla \mathbf{u}_{b}\right) \cdot \mathbf{n} d S \\
& -\int_{\mathcal{E}_{i}} \llbracket \mathbf{u}_{h} \rrbracket\left\{\left\{\mathbf{G}^{T}\left(\mathbf{u}_{h}\right) \nabla_{h} \phi_{h}\right\}\right] \cdot \mathbf{n} d S-\int_{\mathcal{E}_{b}}\left(\mathbf{u}_{h}^{+}-\mathbf{u}_{b}\right)\left\{\left\{\mathbf{G}^{T}\left(\mathbf{u}_{b}\right) \nabla_{h} \phi_{h}^{+}\right\}\right] \cdot \mathbf{n} d S \\
& +\int_{\mathcal{E}_{i}} \llbracket \phi_{h} \rrbracket \boldsymbol{\delta}\left(\mathbf{u}_{h}\right) \cdot \mathbf{n} d S+\int_{\mathcal{E}_{b}} \phi_{h}^{+} \boldsymbol{\delta}_{b}\left(\mathbf{u}_{h}^{+}, \mathbf{u}_{b}\right) \cdot \mathbf{n} d S
\end{aligned}
$$

where $\mathbf{G}\left(\mathbf{u}_{h}\right)=\partial \mathcal{F}_{v}\left(\mathbf{u}_{h}, \nabla_{h} \mathbf{u}_{h}\right) / \partial\left(\nabla_{h} \mathbf{u}_{h}\right)$ is the so-called homogeneity tensor, so that the viscous fluxes may be written as $\mathcal{F}_{v}\left(\mathbf{u}_{h}, \nabla_{h} \mathbf{u}_{h}\right)=\mathbf{G}\left(\mathbf{u}_{h}\right) \nabla_{h} \mathbf{u}_{h}$. Following the approach proposed by Hartmann and Houston [28], the penalty functions are written as

$$
\begin{array}{r}
\boldsymbol{\delta}\left(\mathbf{u}_{h}\right)=\sigma\left\{\left\{\mathbf{G}\left(\mathbf{u}_{h}\right)\right\}\right\} \llbracket \mathbf{u}_{h} \rrbracket \\
\left.\boldsymbol{\delta}_{b}\left(\mathbf{u}_{h}^{+}, \mathbf{u}_{b}\right)=\sigma_{b}\left\{\mathbf{G}\left(\mathbf{u}_{b}\right)\right\}\right\}\left(\mathbf{u}_{h}^{+}-\mathbf{u}_{b}\right)
\end{array}
$$

where $\sigma$ and $\sigma_{b}$ are called the penalty coefficients. In this work, the optimal penalty coefficients derived in [29] in the context of hybrid meshes are adopted. In the framework of $p$-adaptive methods, the polynomial degree $p$ in each of the two elements sharing an interior face $f=\partial K^{+} \cap \partial K^{-}$might take on different values. In this case, the penalty 
coefficient is given by $\sigma=\eta \max \left(c_{K^{+}}, c_{K^{-}}\right)$, where, if we consider hexahedral meshes, the function $c_{K}$ is defined for each mesh element $K$ by

$$
c_{K}=\frac{\left(p_{K}+1\right)^{2}}{V_{K}}\left(\frac{1}{2} \sum_{f \in \partial K \backslash \mathcal{E}_{b}} A_{f}+\sum_{f \in \partial K \cap \mathcal{E}_{b}} A_{f}\right)
$$

in which $p_{K}$ is the polynomial degree associated with element $K, V_{K}$ its volume, and $A_{f}$ the surface of a face $f \in \partial K$. On a boundary face, the penalty coefficient is simply defined by $\sigma_{b}=\eta c_{K^{+}}$. The constant $\eta$ is taken equal to one for the simulations presented in this work.

The integrals in Eqns. (5) and (7) are computed by means of the Gauss-Legendre quadrature with $q=p+1+m$ points in each space direction, where $m$ depends on the test case considered. As regards time integration, an explicit third-order accurate Runge-Kutta method is employed.

The effect of the subgrid scales can be approximated by a closure term that depends only on the resolved field, $\mathcal{L}_{s g s}\left(\mathbf{u}, \mathbf{u}_{h}, \phi_{h}\right) \approx \mathcal{L}_{m}\left(\mathbf{u}_{h}, \phi_{h}\right)$. The compressible LES formalism of [46] is used here, which consists in introducing a SGS flux vector of the form,

$$
\mathcal{F}_{m}=\left(0, \tau^{\mathbf{s g s}},-\mathbf{q}^{\mathbf{s g s}}\right)^{T}
$$

Using the eddy-viscosity assumption, the SGS stress tensor $\tau^{\text {sgs }}$ is written as

$$
\tau_{i j}^{\mathrm{sgs}}=\rho_{h} v_{t}\left(2 S_{i j}-\frac{2}{3} S_{k k} \delta_{i j}\right)
$$

where $\rho_{h}$ is the resolved density, $S_{i j}$ are the components of the resolved rate-of-strain tensor $\mathbf{S}$, and $v_{t}$ the turbulent eddy viscosity. The SGS heat-flux vector is given by

$$
q_{i}^{\mathrm{sgs}}=-\rho_{h} C_{p} \frac{v_{t}}{P r_{t}} \frac{\partial T_{h}}{\partial x_{i}}
$$

where $T_{h}$ denotes the resolved temperature and $P r_{t}$ is the turbulent Prandtl number, assumed to be constant and equal to 0.6 .

In this work, two SGS models are considered, the Smagorinsky model [73] and the Wall-Adapting Local Eddy-viscosity (WALE) approach proposed by Nicoud and Ducros [63].

\section{The Smagorinsky model}

For the Smagorinsky model, $v_{t}$ is expressed as

$$
v_{t}=\left(C_{s} \Delta\right)^{2}|\mathbf{S}| ;|\mathbf{S}|=\sqrt{2 S_{i j} S_{i j}}
$$

In (14), $C_{s}$ is the model constant, set in this work to $C_{s}=0.1$, and $\Delta$ is the local filter width defined in terms of the volume of the element, $V_{K}$, and the local polynomial degree, $p_{K}$, namely, as $\Delta=\sqrt[3]{V_{K}} /\left(p_{K}+1\right)$. 
The WALE model

This model is based on a tensor invariant and is able to represent the proper scaling at the wall, $v_{t}=O\left(y^{3}\right)$. In the WALE approach, the eddy viscosity $v_{t}$ is expressed as

$$
v_{t}=\left(C_{W} \Delta\right)^{2} \frac{\left(S_{i j}^{d} S_{i j}^{d}\right)^{\frac{3}{2}}}{\left(S_{i j} S_{i j}\right)^{\frac{5}{2}}+\left(S_{i j}^{d} S_{i j}^{d}\right)^{\frac{5}{4}}}
$$

where $S_{i j}^{d}$ is defined by

$$
S_{i j}^{d}=\frac{1}{2}\left(g_{i j}^{2}+g_{j i}^{2}\right)-\frac{1}{3} \delta_{i j} g_{k k}^{2}
$$

and it represents the symmetric part of the tensor $g_{i j}^{2}=g_{i k} g_{k j}$, where $g_{i j}=\partial u_{h, i} / \partial x_{j}$. The filter width $\Delta$ is defined for each element as above, and the model constant is set to $C_{W} \leq 0.6$ as recommended in [63].

Based on the formalism laid out above, the model term $\mathcal{L}_{m}\left(\mathbf{u}_{h}, \phi_{h}\right)$ is discretized using the same scheme employed to discretize the viscous fluxes (see Eqn. (7)). For more details on the DG-LES formalism used in this work, the reader is referred to [15, 20].

\section{The DG-VMS formulation}

In VMS we assume that the effect of the unresolved scales on the largest resolved scales is negligible and thus the effect of the SGS model is confined to a range of small resolved scales. This is obtained in the DG-VMS framework by splitting the polynomial solution space $\mathcal{V}_{h}^{p}$ into a low-order component $\mathcal{V}^{l} \equiv \mathcal{V}_{h}^{p_{L}} \subseteq \mathcal{V}_{h}^{p}$, associated with the large scales, and a high-order component $\mathcal{V}^{s} \equiv \mathcal{V}_{h}^{p} \backslash \mathcal{V}^{l}$ representing the small resolved scales, where $p_{L}$ is called the scale-partition parameter. In this work, the SGS model term is computed from the full resolved field, as proposed in [15], and the effect of the SGS model is removed from all scales belonging to $\mathcal{V}^{l}$ by enforcing $\mathcal{L}_{m}\left(\mathbf{u}_{h}, \phi_{h}\right)=0, \forall \phi_{h} \in \mathcal{V}^{l}$.

\section{Choice of numerical flux based on TGV simulations at $R e=20000$}

Recent research has highlighted the deficiencies of the LLF scheme for the simulation of low-Mach number turbulence in the context of under-resolved DG simulations of the Euler equations [54, 55] and in studies of 2D grid turbulence [53], based on a no-model, or implicit LES (ILES), approach.

In this section we focus on the effect of the flux functions defined in Sec. 3 on the different components of the kinetic energy (k.e.) balance equation when a SGS modelling approach is employed at high (but finite) Reynolds number. To this end, we perform DG simulations of the TGV configuration at $R e=20000$ and $M_{0}=0.1$, based on the standard LLF and Roe schemes and their respective modified versions. For comparison, the ILES approach is also considered. 


\subsection{The Taylor-Green vortex configuration}

The TGV model problem constitutes a benchmark test case for the evaluation of numerical schemes and LES modelling approaches. It represents a transitional flow in a tri-periodic box defined in the domain $\Omega=[-\pi, \pi]^{3}$.

The initial condition is given by a solenoidal velocity field with components $(u, v, w)$ and a pressure field $p$ satisfying Poisson equation, namely

$$
\begin{aligned}
& u(\mathbf{x}, 0)=\sin (x) \cos (y) \cos (z) \\
& v(\mathbf{x}, 0)=-\cos (x) \sin (y) \cos (z) \\
& w(\mathbf{x}, 0)=0 \\
& p(\mathbf{x}, 0)=\frac{1}{\gamma M_{0}^{2}}+\frac{1}{16}(\cos (2 x)+\cos (2 y))(\cos (2 z)+2)
\end{aligned}
$$

where $\gamma$ is the adiabatic index and $M_{0}$ the Mach number. The density at $t=0$ is assumed to be constant and equal to $\rho(\mathbf{x}, 0)=1$.

The flow is characterised by a transient phase in which the initial large-scale vortices break up into smaller vortices generating a 3D turbulent energy cascade and reaching a peak of dissipation after which turbulence decays freely. The rate at which the k.e. decays during this final stage is provided by the k.e. balance equation

$$
-\frac{d E_{k}}{d t}=\varepsilon+\varepsilon_{c}
$$

in which $E_{k}$ is the volume-averaged k.e., $\varepsilon$ is the viscous dissipation rate and $\varepsilon_{c}$ is the work done by the thermodynamic pressure. These three quantities are defined as follows

$$
\begin{aligned}
E_{k} & =\frac{1}{\rho_{0}|\Omega|} \int_{\Omega} \rho \frac{\mathbf{v} \cdot \mathbf{v}}{2} d \Omega \\
\varepsilon & =\frac{1}{\rho_{0}|\Omega|} \int_{\Omega} 2 \mu \mathbf{S}^{(d)}: \mathbf{S}^{(d)} d \Omega \\
\varepsilon_{c} & =\frac{1}{\rho_{0}|\Omega|} \int_{\Omega}-p \nabla \cdot \mathbf{u} d \Omega
\end{aligned}
$$

where $\mu$ is the molecular viscosity, $\rho_{0}$ is the volume-averaged density, and $\mathbf{S}^{(d)}=$ $\mathbf{S}-\frac{1}{3}(\nabla \cdot \mathbf{v}) \mathbf{I}$ denotes the deviatoric part of the rate-of-strain tensor. Note, that the enstrophy

$$
\xi=\frac{1}{\rho_{0}|\Omega|} \int_{\Omega} \rho \frac{\omega \cdot \omega}{2} d \Omega
$$

is directly related to the viscous dissipation by the relation $\varepsilon=2 \frac{\mu}{\rho_{0}} \xi$. 


\begin{tabular}{lcccccc}
\hline Simulation & Order & $q$ & \# Elt. & \# DOFs & SGS Model & $\Delta t$ \\
\hline VMS-36P6 & 7 & 9 & $36^{3}$ & $252^{3}$ & VMS & $5 \cdot 10^{-4}$ \\
ILES-36P6 & 7 & 9 & $36^{3}$ & $252^{3}$ & - & $5 \cdot 10^{-4}$ \\
\hline
\end{tabular}

Table 1: TGV configuration at $R e=20000$. Details of DG-LES simulations performed.
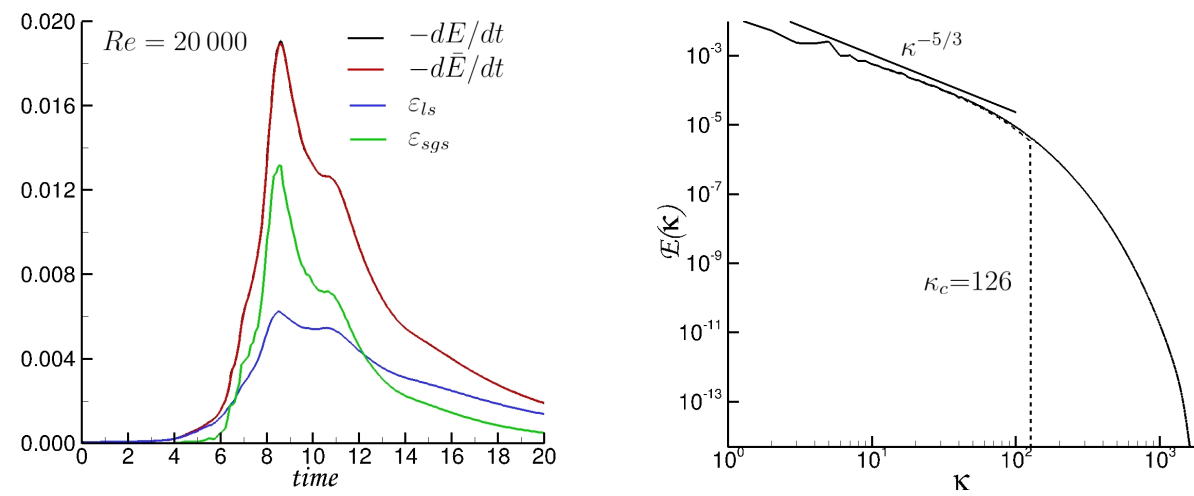

Figure 1: TGV configuration at $R e=20000$. Left: dissipation components of k.e. balance Eqn. (22). Right: Fourier spectra of DNS data at $R e=20000$. The vertical dashed line corresponds to the cut-off wavenumber used to filter the DNS data for the selected resolution.

\subsection{DG-LES simulations of the TGV problem at $R e=20000$}

In this section, the results from the DG simulations are reported and compared to DNS data. The reference DNS has been performed using the sixth-order finitedifference incompressible solver Incompact3D on a grid composed of $3456^{3}$ nodes $[19,44]$.

A very coarse resolution is considered in the LES, corresponding to a total number of DOFs of about $252^{3}$. This amounts to approximately $1 / 14^{3}$ of the number of DOFs of the reference DNS. The polynomial degree employed in these simulations is $p=6$ and the number of quadrature points used to perform the integrals $q=9$. The computational grid is composed of 36 cubic elements in each direction of space. The details of the DG simulations performed are summarised in Table 1.

\subsection{LES kinetic energy balance equation}

In an LES, the k.e. balance equation involves an additional term, $\varepsilon_{s g s}$, called the SGS dissipation, namely,

$$
-\frac{d \bar{E}_{k}}{d t}=\varepsilon_{l s}+\varepsilon_{s g s}+\varepsilon_{c}
$$

where $\bar{E}_{k}$ represents the k.e. of the resolved velocity field.

The term, $\varepsilon_{l s}$, is computed from the expression (19) using the resolved velocity field and is now called the large-scale (LS) dissipation. The LS dissipation is related to the resolved enstrophy by $\varepsilon_{l s}=2 \frac{\mu}{\rho_{0}} \bar{\xi}$, where $\bar{\xi}$ is computed from (21) by using the resolved 
vorticity. The SGS dissipation, $\varepsilon_{s g s}$, on the other hand, accounts for the dissipation that should be provided by the unresolved scales and has to be modelled. Finally, if the Mach number is low, the compressibility term, $\varepsilon_{c}$, is expected to be negligible.

The time-evolution of the different components involved in the k.e. balance equation (22) is shown in the left panel of Fig. 1. These quantities have been computed from the reference DNS by applying a sharp cut-off filter in Fourier space, with cut-off wavenumber equal to half the number of DOFs considered in the LES simulations, i.e. $k_{c}=126$. For reference, the k.e. dissipation from the unfiltered DNS field, $-\frac{d E_{k}}{d t}$, is also shown, which is almost identical to the filtered DNS k.e. dissipation, $-\frac{d \bar{E}_{k}}{d t}$. The right panel of Fig. 1 shows the energy spectra of the DNS at $t=14$ (in normalised time units) as well as the cut-off wavenumber $k_{c}=126$. We can see from the leftward plot that, for this particular combination of coarse resolution and high Reynolds number, the associated SGS dissipation is dominant over its LS counterpart during the turbulent breakdown. This represents a challenging situation in which the SGS model will play a primary role in the LES simulation.

\subsection{Simulation assessment strategy}

In order to assess the quality of our simulations, the time evolution of $\varepsilon_{l s}, \varepsilon_{s g s}, \varepsilon_{c}$, and $-d \bar{E}_{k} / d t$, will be monitored. The derivatives involved in the definition of $\varepsilon_{l s}$ and $\varepsilon_{c}$, Eqns. (19) and (20), respectively, are computed from the DG-LES solution using differential operators which are consistent with the numerical scheme. By consistent, we mean that the differential operator takes into account the jumps across the element interfaces via appropriate lifting operators [5].

Particular focus is placed on the LS dissipation, $\varepsilon_{l s}$, as this quantity is very sensitive to the amount of SGS and numerical dissipation present in the computation. This is in contrast with the k.e. dissipation, $-d \bar{E}_{k} / d t$, which, as will be seen below, appears to be much less affected by the numerics. Note as well that the evolution of the enstrophy (not shown in this study) is equivalent to that of the LS dissipation scaled by a factor $\rho_{0} / 2 \mu$.

As mentioned above, the LS dissipation is computed from Eqn. (19), using the DG-LES velocity field. This quantity will be compared with that obtained from the DNS data. To this end, the DG-projection filter associated with the employed $h p$ discretization is applied to the DNS data. The procedure followed to define this filtering operation is described in detail in [58]. Note however that the levels of $\varepsilon_{l s}$ resulting from this filtering procedure can be very different from those displayed by $\varepsilon_{l s}$ in Fig. 1 . Indeed, as shown in [58], the (discontinuous) DG-filter is not equivalent to a sharp cut-off in Fourier space. It is in fact not possible to define a global transfer function in Fourier space from the inherently local DG-projection operator.

As regards the SGS dissipation, this contribution is computed from Eqn. (22) by writing,

$$
\varepsilon_{s g s}=-\frac{d \bar{E}_{k}}{d t}-\varepsilon_{l s}-\varepsilon_{c}
$$

We note that in the framework of an LES simulation, the SGS component, $\varepsilon_{s g s}$, can be interpreted as the sum of two contributions. The first results from the dissipation provided by the SGS model, that we denote by $\varepsilon_{m o d}$, and the second from the dissipation 
introduced by the employed numerical scheme, which will be referred to as $\varepsilon_{\text {num }}$, such that $\varepsilon_{\text {sgs }}=\varepsilon_{\text {mod }}+\varepsilon_{\text {num }}$.

In the case of an ILES simulation, it is obvious that the first contribution is equal to zero and the SGS dissipation is solely due to the dissipation introduced by the numerical scheme, i.e. $\varepsilon_{s g s}=\varepsilon_{n u m}$. On the other hand, when an eddy-viscosity approach is employed in a model-based LES simulation, $\varepsilon_{m o d}$ can be computed directly from Eqn. (19) by replacing $\mu$ by the expression of the eddy viscosity $\mu_{t}$, namely,

$$
\varepsilon_{\text {mod }}=\frac{1}{\rho_{0}|\Omega|} \int_{\Omega} 2 \mu_{t} \mathbf{S}^{(d)}: \mathbf{S}^{(d)} d \Omega
$$

in which $\mathbf{S}^{(d)}$ is computed from the resolved velocity field. In this case, the contribution of the numerical dissipation to the k.e. dissipation could be derived from $\varepsilon_{\text {num }}=$ $-\frac{d \bar{E}_{k}}{d t}-\varepsilon_{l s}-\varepsilon_{\text {mod }}-\varepsilon_{c}$.

In the context of a VMS-like approach, finding an explicit expression for $\varepsilon_{m o d}$ becomes more involved. The reason is that in this case the modelled SGS stress tensor is only applied to a given range of modes, defined by the scale-partition parameter $p_{L}$ (see Sec. 4). A way to proceed would thus be to compute the dissipation due to the model term directly from the modelled SGS stress tensor, $\tau^{\text {sgs }}$, by writing

$$
\varepsilon_{\text {mod }}=\frac{1}{|\Omega|} \int_{\Omega} \overline{\mathbf{v}}^{s} \cdot{\overline{\left(\nabla \cdot \tau^{s g s}\right.}}^{s} d \Omega, \quad \text { with } \quad \tau^{s g s}=2 \mu_{t} \mathbf{S}^{(d)}
$$

in which $\mathbf{v}$ is the resolved velocity vector and the operator $\overline{(\cdot)}^{s}$ represents the projection of a quantity onto the polynomial space associated with the high-order modes, given by $\mathcal{V}^{s} \equiv \mathcal{V}_{h}^{p} \backslash \mathcal{V}^{p_{L}}$ (see Sec. 4). This implies that an expression for $\varepsilon_{m o d}$ of the form of Eqn. (24) above is not readily available.

Note as well that, in a DG-LES, expression (25) is not strictly equivalent to Eqn. (24). Observe that the computation of $\overline{\left(\nabla \cdot \tau^{s g s}\right)}$, involves two contributions, one from a volume integral and another one from a surface integral. The latter contribution vanishes if the jumps in the solution across element interfaces are negligible. This will rarely be the case in an LES. It is well-known that these surface integrals play, via the jumps, a stabilising role in the simulation [11], and therefore contribute to numerical dissipation. We thereby see that, in the framework of DG-VMS, separating the contributions due to purely physical modelling effects from those due to numerical dissipation in a consistent manner is not straightforward.

The philosophy adopted in this research is thus to consider that our DG-LES solution should follow as closely as possible the physics underlying the k.e. balance equation (22). The three resolved components $-\frac{d E_{k}}{d t}, \varepsilon_{l s}$, and $\varepsilon_{c}$ are then computed from the DG-LES solution, whereas any other form of contribution to $-\frac{d E_{k}}{d t}$, whether it comes from the modelling or from the numerics, will contribute to the SGS dissipation component and is gathered in $\varepsilon_{s g s}$.

We further remark, that when a low-dissipative numerical flux, i.e. $\alpha \leq 0.1$, is considered in conjunction with a high value of the polynomial degree, the effects of numerical dissipation in our DG-VMS will be considerably reduced. In this case, it might not be unrealistic to consider that most of the SGS dissipation can be attributed to the SGS model, and thus that $\varepsilon_{s g s} \approx \varepsilon_{\text {mod }}$. 

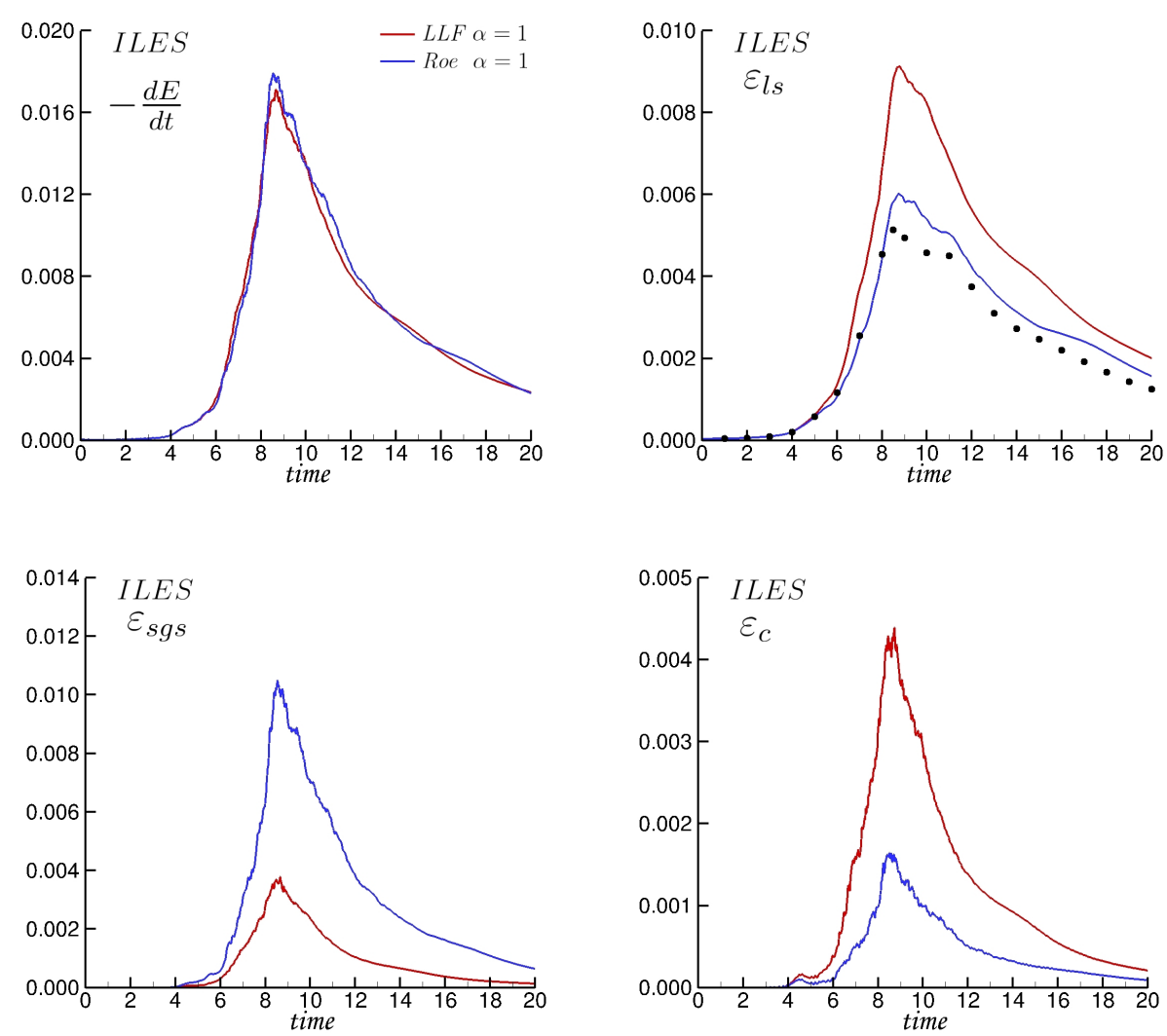

Figure 2: TGV configuration at $R e=20000$. Time-evolution of the dissipation components for the ILES36P6 simulations using the LLF and Roe fluxes. The symbols represent the reference DG-filtered DNS.

Finally, we point out that in the context of low-Mach-number flows, the compressibility term, $\varepsilon_{c}$, is expected to be negligible. We will see, however, that this term can actually take on very significant values, and is strongly affected by the numerical discretization. It could be argued, that there is in principle no guarantee that the compressibility term computed from the DG-filtered DNS data is zero, and thus that a negligible value of $\varepsilon_{c}$ cannot be taken as a quality criterion. We will see however, from the results presented in Secs. 5.4.1 to 5.4.3, that there is a clear correlation between the quality of the DG results, in terms of a closer agreement with the reference LS dissipation, $\varepsilon_{l s}$, and the fact that $\varepsilon_{c}$ be small.

\subsubsection{ILES simulations: effect of numerical flux}

Figure 2 compares the evolution of the dissipation components obtained from the ILES simulations using the standard LLF and the Roe schemes, i.e. $\alpha=1$, for a DG $p=6$ discretization.

It is obvious from these plots that $-d \bar{E}_{k} / d t$ is nearly unaffected by the amount of dissipation introduced by the numerical flux. In particular, the peak of dissipation does 
not change much in position nor in intensity for the two cases considered. This is in contrast with $\varepsilon_{l s}$, which, as we can see from Fig. 2, presents a very different behaviour for the two schemes considered.

More specifically, the use of the LLF flux leads to an overestimation of the level of $\varepsilon_{l s}$, and an underestimation of the level of $\varepsilon_{s g s}$, with respect to the DG-filtered DNS data. The use of the Roe scheme greatly improves the results, with levels of LS dissipation much closer to the reference data, though still above the reference values, by about $20 \%$ at the location of the peak. This, somehow counter-intuitive, behaviour appears to be directly related to the non-negligible values of the compressibility term $\varepsilon_{c}$ found in these simulations. As shown in Fig. 2, $\varepsilon_{c}$ represents about $20 \%$ and $9 \%$ of the k.e. dissipation peak for the simulations based on the LLF and the Roe schemes, respectively. In the context of this low-Mach-number flow, it is fair to interpret these non-negligible values of $\varepsilon_{c}$ as numerical errors. The numerical experiments performed in the following sections show that the cause of this unacceptable behaviour is linked to numerical errors introduced via the upwind component of the Riemann solver, also pointed out in [54]. The magnitude of these errors is related to the magnitude of the jumps in the DG solution, and therefore to the degree of under-resolution in the simulation. We will see below, that the use of a SGS model in combination with a low-dissipative flux function can mitigate this problem.

\subsubsection{DG-VMS simulations: effect of numerical flux}

Figures 3 and 4 show the time evolution of the dissipation components obtained from DG-VMS simulations based on the LLF and Roe fluxes, respectively. The effect of the upwind term has been investigated by considering different values of the upwind scaling parameter, namely, $\alpha=\{1,0.2,0.1,0.05\}$. We note that removing completely the upwind dissipation, i.e. setting $\alpha=0$, leads to divergence of the solution. The reason for this behaviour is not fully understood, although it seems to be related to the spectral behaviour of the (physical or ideal) SGS dissipation near the grid cutoff, which VMS cannot exactly replicate. This has been pointed out in [58] (Sec. 2.2, page 111). It appears from the outcome of the aforementioned study that a minimum amount of numerical dissipation is required to stabilise the simulation.

In these simulations, the scale-partition parameter is set to $p_{L}=3$, for which the partition ratio, defined by $\left(p_{L}+1\right) /(p+1)$, is 0.57 . This value is within the recommended range provided in the literature [30].

The first conclusion emerging from these results is that the SGS model has the ability to reduce the numerical errors introduced by the numerical flux. This is visible from the smaller magnitude of the differences observed in the results when the standard versions of the LLF and the Roe schemes are used, in contrast with the significant change exhibited by the ILES results (see Fig. 1). This is a consequence of the smoothing effect that the SGS model has on the solution, which results in weaker jumps, as already pointed out in [20].

The most important differences are observed when $\alpha=1$ in the peak values of $\varepsilon_{c}$ and $\varepsilon_{s g s}$, being, respectively, about $78 \%$ higher and $9 \%$ lower for the standard LLF with respect to the standard Roe-based simulation. This is consistent with the trends found in the ILES computations, although the differences are now much less marked. 

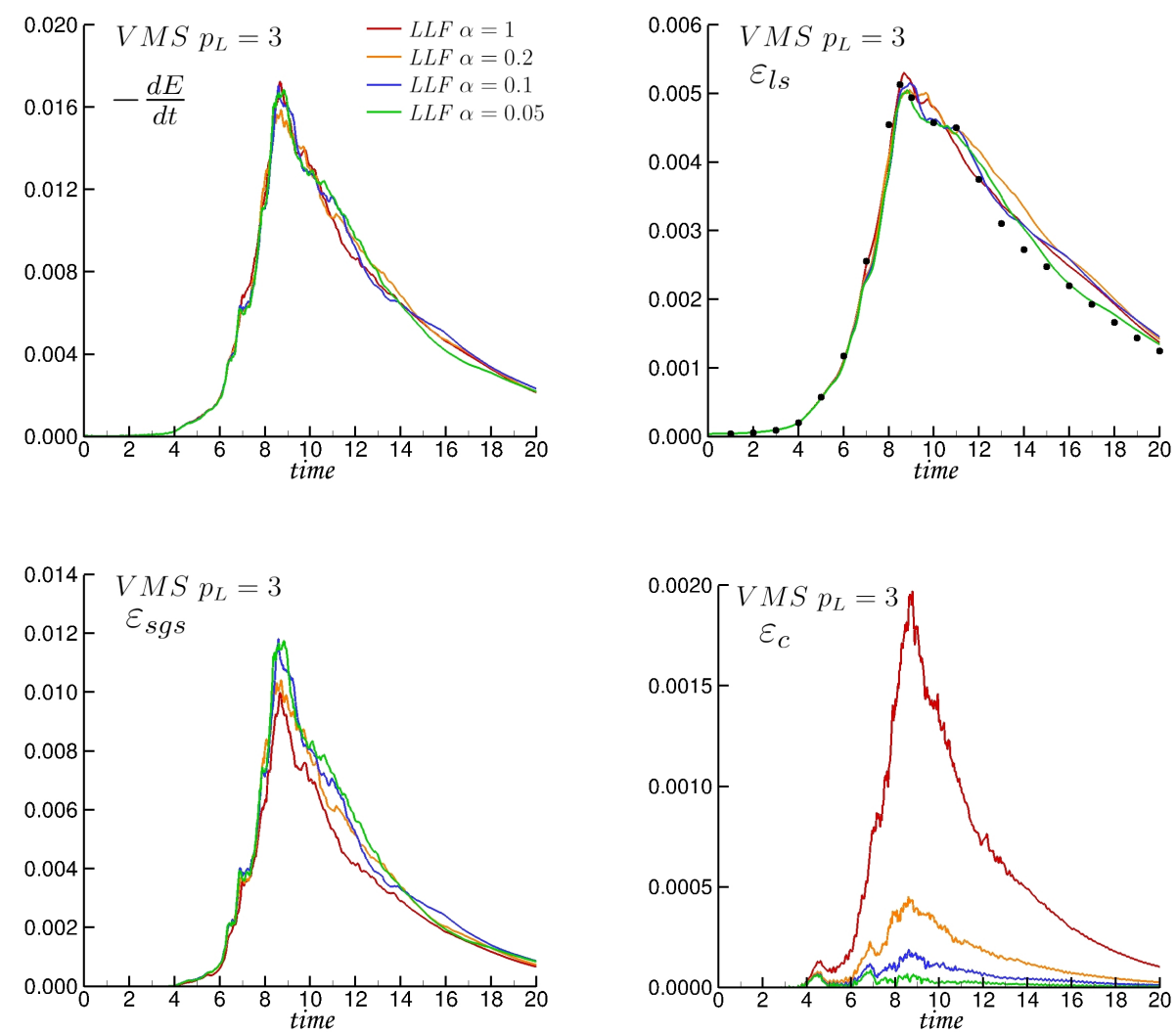

Figure 3: TGV configuration at $R e=20000$. Time-evolution of the dissipation components for the VMS36P6 simulations using $p_{L}=3$ and the LLF scheme with different values of the scaling parameter $\alpha$. The symbols represent the reference DG-filtered DNS.

From these plots, it appears that the best match with the reference DG-filtered DNS data is obtained for the values of $\alpha=0.1$ and 0.05 , for the LLF, and $\alpha=0.1$ for the Roe scheme, for which $\varepsilon_{c}$ is negligible. For these simulations, the levels of SGS dissipation amount to about $68 \%$ of the k.e. dissipation at the peak, very close to the levels shown by the spectrally filtered DNS results shown in Fig. 1, for which the peak value of $\varepsilon_{s g s}$ represents approximately $70 \%$ of the k.e. dissipation at the peak. In the case of the Roe-based ILES simulation, this value represents only $58 \%$ of the k.e. dissipation at the peak, which is consistent with the overestimation of $\varepsilon_{l s}$, and thereby, of the enstrophy $\bar{\xi}$, found for this simulation.

\subsubsection{Comparison of DG-VMS and ILES results and final conclusions}

Finally, Fig. 5 compares the results obtained from the ILES computation based on Roe scheme, and the DG-VMS simulation using a low-dissipative Roe scheme with $\alpha=0.1$, for which the best overall agreement with the reference filtered DNS has been found (see Figs. 2 and 4). We can see that although the curves of k.e. dissipation 

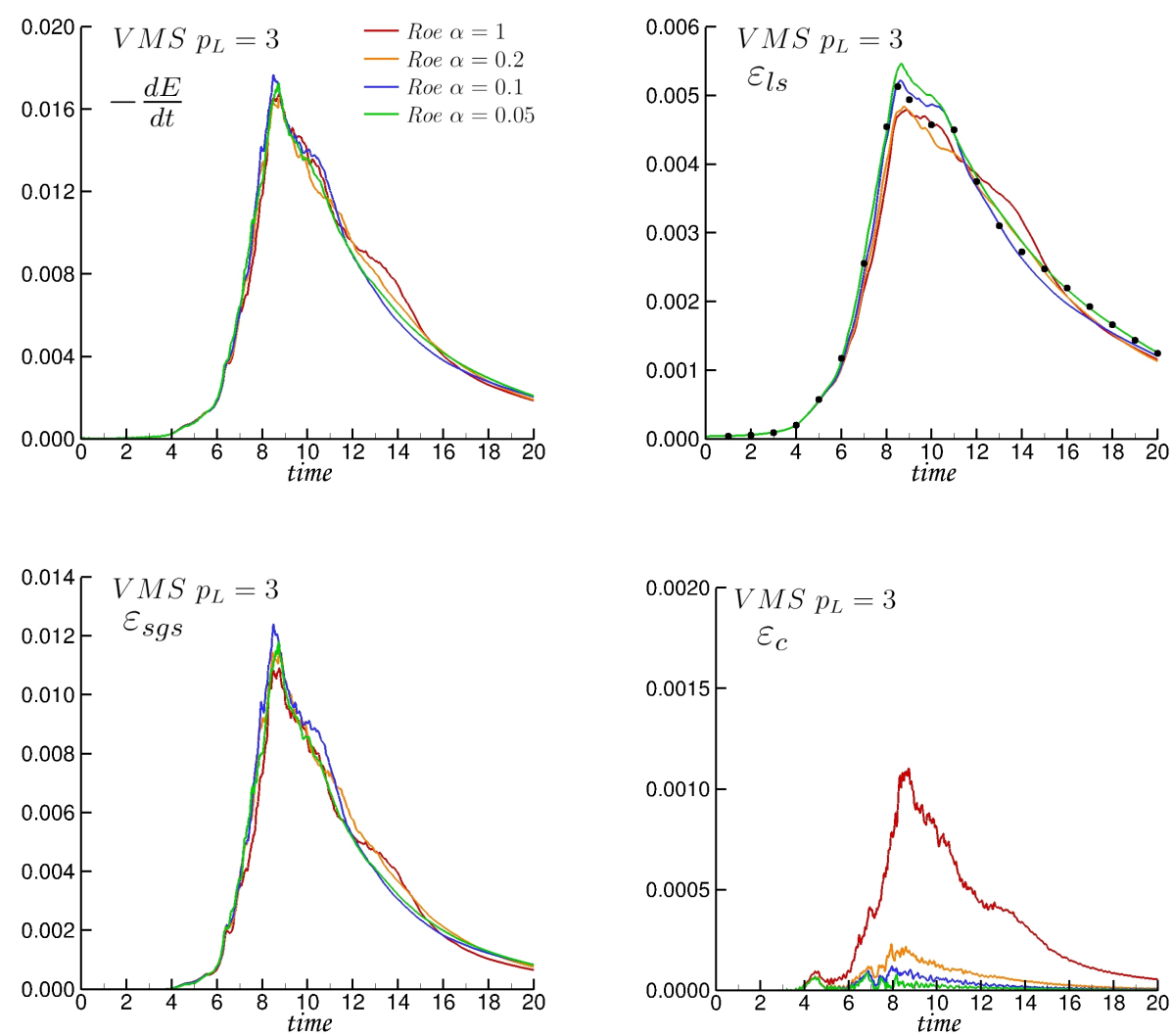

Figure 4: TGV configuration at $R e=20000$. Time-evolution of the dissipation components for the VMS36P6 simulations using $p_{L}=3$ and the Roe scheme with different values of the scaling parameter $\alpha$. The symbols represent the reference DG-filtered DNS.

are very close for both simulations, there are important discrepancies in the three dissipation components that make up the right-hand-side of the k.e. balance equation. In particular, the ILES simulation presents lower values of SGS dissipation and higher values of LS dissipation than for the DG-VMS simulation, as well as a non-negligible compressibility term. This behaviour is not in agreement with the real physics of this problem.

To summarise, it appears from this research that the use of the DG-VMS approach in combination with the least dissipative possible numerical flux leads to the best match with the reference data, with a slight advantage for the low-dissipative Roe scheme over the LLF scheme. We have also put forward the strong influence of the numerical flux function on the no-model DG approach for under-resolved simulations when the Reynolds number is high, and the much more robust behaviour of the VMS approach.

Note that these conclusions are expected to hold in the context of $p$-adaptive simulations, such as the one presented in Sec. 6.5. For this type of simulation, high values of $p$ will be actually selected by the refinement indicator in under-resolved turbulent 

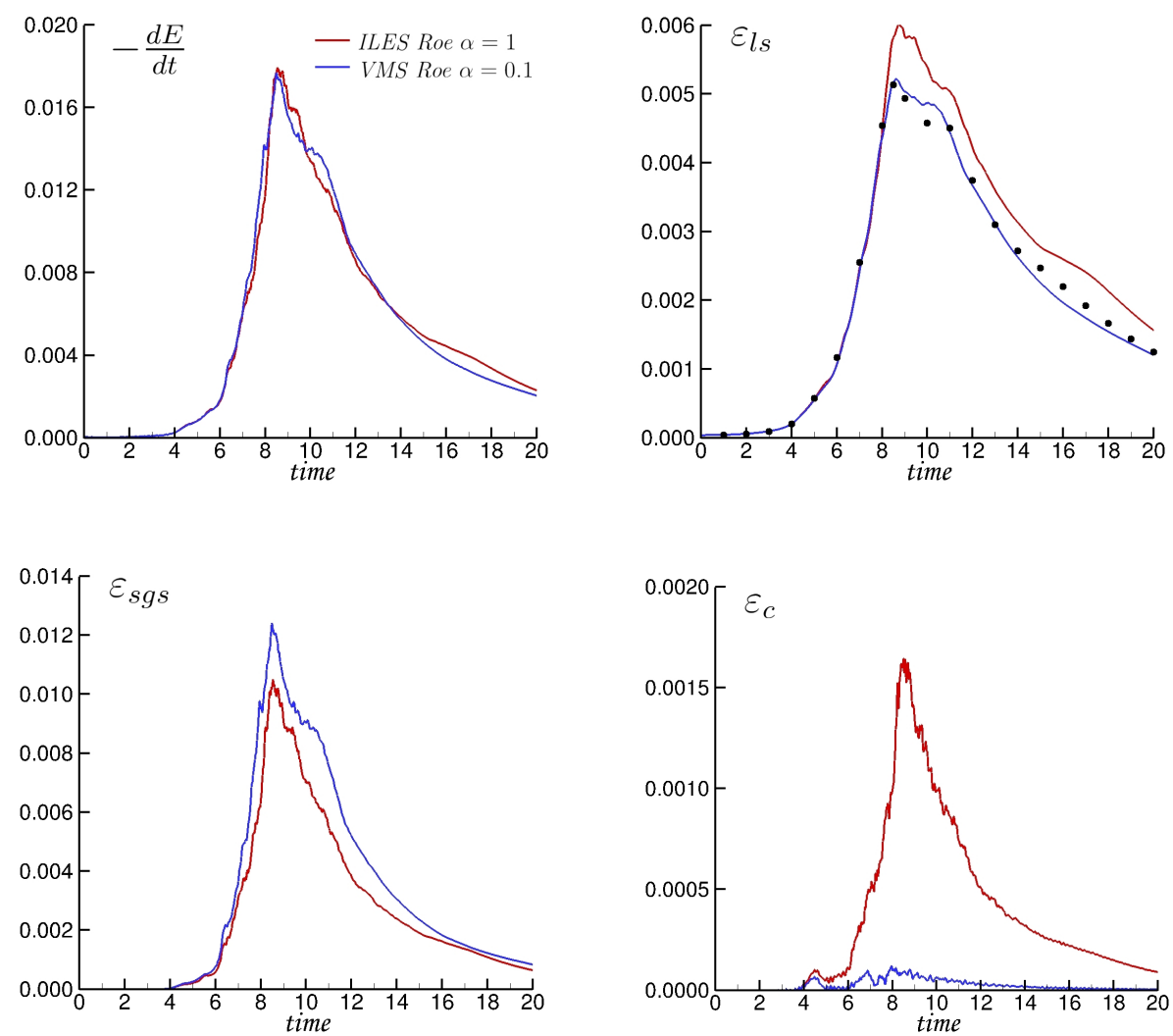

Figure 5: TGV configuration at $R e=20000$. Time-evolution of the dissipation components for the best VMS-36P6 and ILES-36P6 simulations. The symbols represent the reference DG-filtered DNS.

regions. In this situation, we have seen that the DG-VMS approach in combination with a low-dissipative numerical flux performs best. On the other hand, for well-resolved laminar regions with corresponding low values of the polynomial degree, the ILES approach is supposed to perform best. We will see however from the results obtained in the following sections that the combined use of the quasi-central flux and the DG-VMS method based on a high value of the scale-partition ratio $\left(p_{L}+1\right) /(p+1)$ (small fraction of small scales) leads to equally good results.

Finally, we would like to highlight the importance of examining each of the terms involved in the k.e. balance equation, rather than focusing on the analysis of an isolated quantity such as the k.e. dissipation, or even the enstrophy alone. Indeed, we have seen from this study that a successful LES simulation should be able to yield accurate results for $\varepsilon_{l s}, \varepsilon_{s g s}$ and $\varepsilon_{c}$, separately. 


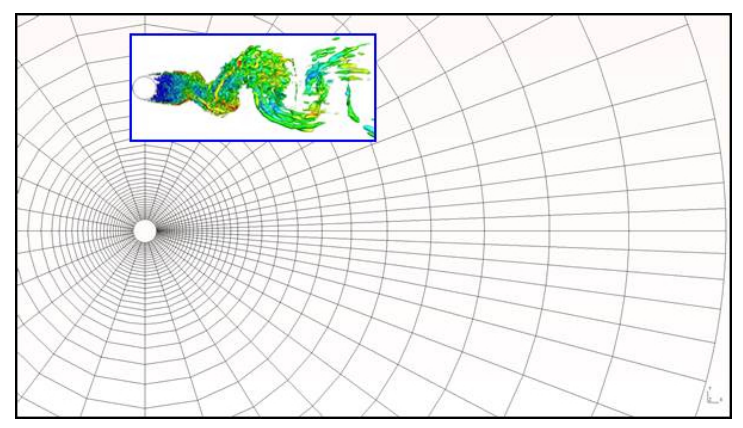

Figure 6: Cylinder flow at $R e=3900$ : Computational grid in the far-wake region.

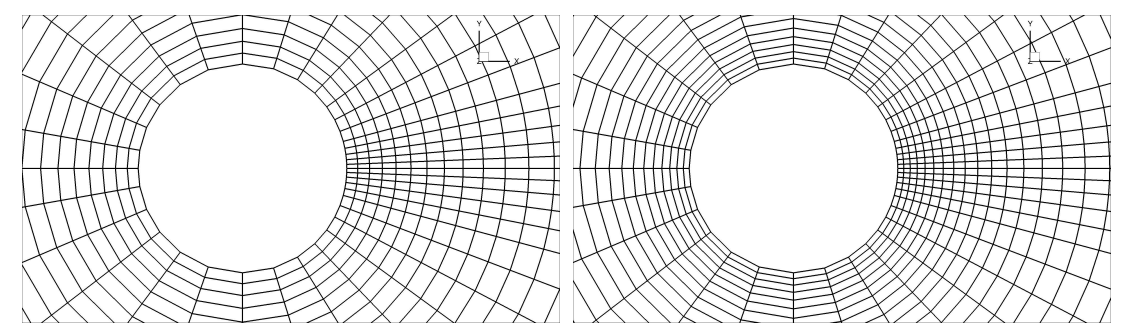

Figure 7: Detail of O-type mesh used in the cylinder simulations. Left: grid used for $R e=3900$ and 20000. Right: grid used for $R e=140000$.

\section{LES of the sub-critical flow past a circular cylinder}

The study of flows past cylinders is of relevance to many engineering applications, such as noise prediction in aircraft landing gears. A characteristic feature of bluff body aerodynamics is the appearance of a large-scale vortex shedding in the near wake which can lead to flow-induced sound and vibration of the structure. The ability to accurately predict this phenomenon across a wide range of Reynolds numbers using numerical methods is especially challenging. In the context of high-order methods, the geometrical representation of the cylinder adds an extra level of complexity due to the necessity to use large curved elements around the wall, when the order of the polynomial approximation is increased ( $p$-refinement).

The sections that follow report the results from the DG-LES simulations of the cylinder flow at $R e=3900,20000$ and 140000 . In this sub-critical regime, the boundary layer separates laminarly from the cylinder surface and transition to turbulence takes place in the free-shear layer. For the lowest Reynolds, three different approaches are considered: no-model, standard LES based on the WALE model, and VMS based on the Smagorinsky model. For 20000 and 140 000, only the VMS approach is considered.

A large number of LES simulations of the circular cylinder configuration at $R e=$ 3900 can be found in the literature, which employ different numerical methods and SGS modelling approaches. We can cite for example the work in [77, 51, 56, 66, 9] using second-order FV methods. Model-based LES simulations of this configuration using 
Table 2: Computational details of the DG simulations of the cylinder flow configuration. The time step $\Delta t$ is normalised by the cylinder diameter $D$ and the free-stream velocity $U_{c}$. The number of vortex shedding periods $T_{v s}$ over which statistical averaging is performed is denoted by $T_{a v g} / T_{v s}$.

\begin{tabular}{llcclcccc}
\hline Re & SGS model & Order & $q$ & Flux & \# Mdofs & $\frac{\Delta r}{D(p+1)}$ & $\Delta t\left(\frac{U_{c}}{D}\right)$ & $\frac{T_{a v g}}{T_{v s}}$ \\
\hline 3900 & WALE & 5 & 9 & LLF $(\alpha=0.1)$ & 2.59 & $10^{-2}$ & $2.0 \cdot 10^{-4}$ & 100 \\
3900 & VMS $\left(p_{L}=3\right)$ & 5 & 9 & $\operatorname{LLF}(\alpha=0.1)$ & 2.59 & $10^{-2}$ & $3.0 \cdot 10^{-4}$ & 150 \\
3900 & - & 5 & 9 & $\operatorname{Roe}(\alpha=1.0)$ & 2.59 & $10^{-2}$ & $5.0 \cdot 10^{-4}$ & 150 \\
\hline 20000 & \multirow{2}{*}{$\operatorname{VMS}\left(p_{L}=1\right)$} & 5 & 9 & $\operatorname{LLF}(\alpha=0.1)$ & 2.59 & $10^{-2}$ & $2.0 \cdot 10^{-4}$ & 95 \\
\hline 140000 & $\operatorname{VMS}\left(p_{L}=1\right)$ & 5 & $4-10$ & $\operatorname{Roe}(\alpha=0.1)$ & 4.98 & $4 \cdot 10^{-3}$ & $1.5 \cdot 10^{-4}$ & 71 \\
\hline
\end{tabular}

high-order discontinuous methods have been reported by Beck et al. [7] using the standard LES and the VMS approach based on the Smagorinsky model, and by Ferrer [22] using an ILES approach based on an DG-Fourier method supplemented by a Spectral Vanishing Viscosity (SVV) method in the Fourier direction for stabilisation. More recently, Lodato and Chapelier have performed simulations of this configurations using the spectral-difference method in combination with a SGS modelling approach [49].

For the two higher Reynolds numbers, a limited number of LES simulations of this configuration have been, however, reported in the literature. We can cite the work presented in [72, 56, 52] for $R e=20000$ and in $[10,24]$ for $R e=140000$ based on second-order FV methods and different SGS models and grid resolutions.

\subsection{Computational grids and model parameters}

In all simulations an O-type curved hexahedral mesh is considered in a computational domain with radial and spanwise extension of $25 D$ and $\pi D$, respectively. The number of elements in the azimuthal and radial directions is 36 . In the spanwise direction 16 elements are used. This amounts to a total number of elements of 20736 . In the case of the highest Reynolds number, a higher stretching factor is used in a region closer to the cylinder, which leads to a more pronounced clustering of the elements in the vicinity of the wall, with respect to the lower-Reynolds-number case (see Fig. 7). As will be shown below, this is consistent with the shorter length of the recirculation bubble expected and the resolution requirements of the boundary layer at this higher Reynolds number. The focus in this work is therefore on capturing the flow dynamics in the near-wake behind the cylinder, by putting most of the computational effort in this region. The region beyond the recirculation area can thus be considered as under-resolved (see Fig. 6).

The mesh resolution at the wall is $\Delta r=0.05 D$ for the first mesh (Fig. 7 left) and $\Delta r=0.028 D$ for the second (Fig. 7 right).

A uniform polynomial degree $p=4$, is used for the simulations at $R e=3900$ and 20000 , which leads to fifth-order accuracy in space, and an effective resolution at the wall of $\Delta r /(p+1)=0.01 D$. The number of unknowns of the problem is 2.59 million DOFs (Mdofs). For the case at $R e=140000$ a non-uniform distribution of local polynomial degrees is defined based on a recently developed error estimation strategy [59], which leads to a number of unknowns of 4.98 Mdofs. The $p$-adaptation 
strategy used for this simulation is described in detail in Sec. 6.5.1. The polynomial degree associated with the first layer of cells at the wall reaches a value of $p=6$ after two iterations of the $p$-adaptation algorithm, which leads to an effective wall resolution of $\Delta r /(p+1)=4 \cdot 10^{-3} \mathrm{D}$.

Periodicity of the flow is assumed in the spanwise direction, and an isothermal noslip boundary condition is imposed on the cylinder wall. A laminar free-stream flow at Mach number $M=0.2$ is imposed via a non-reflecting condition at the outer boundary of the computational domain, and used to initialise the simulations. After a transient period, a statistically steady (periodic) state is reached, from which the flow statistics are gathered for a sufficient number of vortex-shedding periods, $T_{v s}$, until statistical convergence is reached.

Following from the conclusions of Sec. 5, the low-dissipative versions of the LLF and Roe schemes with $\alpha=0.1$, have been used in the model-based simulations, whereas the standard Roe flux $(\alpha=1)$ is used in the no-model simulation at $R e=3900$. We note that the use of the quasi-central scheme in combination with the no-model approach leads to the simulation blowing up from its initial stage.

The details of the different simulations performed are compiled in Table 2. Note that in the version of the VMS algorithm used in this work the scale-partition parameter $p_{L}$ that sets the limit between large and small scales is constant across the domain. This implies that the SGS model will also be active in the cells covering the laminar boundary layer region. This is, however, an improvement with respect to the standard Smagorinsky model. As mentioned by Lévêque at al. [47], the use of the Smagorinsky model in a VMS framework leads to a reduced amount of SGS dissipation being applied to the large resolved scales, compared to the mono-scale Smagorinsky model.

In the DG-VMS simulations presented in this paper, a partition number $p_{L}=3$ has been used for the lowest Reynolds number $R e=3900$, and $p_{L}=1$ for the two higher Reynolds numbers (see Sec. 4 and [15]). This choice is justified by a recent study carried out by the authors on the spectral properties of the DG-VMS method [61], and by the research reported in [44]. Indeed, one of the main conclusions drawn from [61] is that when the resolution limit falls at the beginning of the dissipation range, which is the case when the Reynolds number is low and/or the resolution is high enough, the energy transfer to mode-numbers $m+1 \leq 0.75(p+1)$ is negligible. This is the case for $R e=3900$, for which we have selected $\left(p_{L}+1\right) /(p+1)=0.8$, consistent with the aforementioned study. As the Reynolds number is increased to $R e=20000$, it is expected that the grid cut-off be shifted back towards the inertial range, which justifies the choice of a lower partition ratio $\left(p_{L}+1\right) /(p+1)=0.4$, implying that a higher number of modes will be affected by the SGS dissipation. Finally, for the highest Reynolds number $R e=140000$, for which the polynomial degree is not constant across the domain, $2 \leq p \leq 6$ the partition ratio varies between $\left(p_{L}+1\right) /(p+1)=0.7$ for the well-resolved regions $(p=2)$ and $\left(p_{L}+1\right) /(p+1)=0.3$ for marginally resolved turbulent regions $(p=6)$.

The outcome of our DG-VMS computations will be compared to the high-resolution LES results obtained by Lysenko et al. in [51] and [52], for $R e=3900$ and $R e=$ 20000 , respectively, considering the same extension of the computational domain $(25 D \times \pi D)$, and Mach number $(M=0.2)$ as in our simulations. The DG-VMS results at $\operatorname{Re}=140000$ are contrasted with those obtained by Breuer [10] using a 
low-dissipation second-order FV method and the Smagorinsky model on a coarse and a fine O-type grids with shorter radial extension $(15 D)$ but with the same span width $(\pi D)$ as that considered in this work. For the two lower Reynolds numbers, the integral flow quantities obtained in [56] using a second-order hybrid FV/FE scheme in combination with the VMS approach are also included for comparison. In addition, the analysed quantities will be compared to the experimental data available in the literature [12, 76, 48, 64, 67].

The following sections provide a detailed description of the results obtained from the DG simulations at the three Reynolds numbers considered.

\subsection{Characteristic flow features of cylinder flow at increasing Reynolds number}

Figure 8 shows a snapshot of the iso-surfaces of the Q-criterion coloured by the Mach number (left panel) as well as the streamlines of the time-averaged flow field (right panel) for the three DG-VMS simulations performed. The most salient feature from these plots is the shrinking of the recirculation bubbles with increasing Reynolds number, which is accompanied by an increasingly intense vortex shedding in the cylinder wake. The plots of the mean velocity fields also reveal the existence of two symmetrical secondary bubbles near the separation point, in accordance with the literature [74, 9, 51, 52]. These vortices are imperceptible for $R e=3900$ and become larger at $R e=20000$. It is worth noting that for the highest Reynolds number, the two small secondary bubbles showing up in our simulation were not observed in the experiments, nor in the simulation by Breuer [10]. These secondary bubbles have also been observed by Karabelas [41], who performed LES of this configuration using an incompressible solver. No clear explanation for this discrepancy with the experiments can be given today, and further research will be necessary to clarify this point.

\subsection{Cylinder flow at $R e=3900$ : discussion of results}

Table 3: Cylinder flow at $R e=3$ 900: integral flow quantities.

\begin{tabular}{lllllllllll}
\hline Method & Model & Mach & Mdofs & $\frac{\Delta r}{D(p+1)}$ & $T_{v s}$ & $C_{d}$ & $C_{l, r m s}$ & $-C_{p, b}$ & St & $\frac{L_{r}}{D}$ \\
\hline Parnaudeau.et al [67] & PIV & - & - & - & - & - & - & - & 0.21 & 1.51 \\
Norberg [64] & HWA & - & - & - & - & 0.98 & $0.04-0.15$ & 0.90 & - & - \\
Moussaed et al. [56] & VMS & 0.1 & 1.46 & $10^{-3}$ & 35 & 0.94 & - & 0.81 & 0.22 & 1.47 \\
Lysenko et al. [51] & TKE & 0.2 & 5.76 & $10^{-3}$ & 150 & 0.97 & 0.09 & 0.91 & 0.21 & 1.67 \\
Present DG- $O$ (5) & WALE & 0.2 & 2.59 & $10^{-2}$ & 100 & 0.96 & 0.09 & 0.82 & 0.21 & 1.69 \\
Present DG- $O$ (5) & VMS & 0.2 & 2.59 & $10^{-2}$ & 150 & 0.99 & 0.16 & 0.89 & 0.21 & 1.49 \\
Present DG- $O(5)$ & - & 0.2 & 2.59 & $10^{-2}$ & 150 & 1.00 & 0.15 & 0.89 & 0.21 & 1.42 \\
\hline
\end{tabular}

Table 3 compiles, together with the present DG results, the simulation results from the second-order FV simulation of Lysenko et al. [51] based on the dynamic $k$-equation eddy-viscosity model (TKE) and $M=0.2$, as well as the PIV and HWA experimental data of Parnaudeau et al. [67] and Norberg [64], respectively ${ }^{1}$. For comparison, the second-order VMS simulation results reported by Moussaed et al. [56] are also included.

${ }^{1} \mathrm{PIV}$ stands for particle-image velocimetry and HWA for hot-wire anemometry 

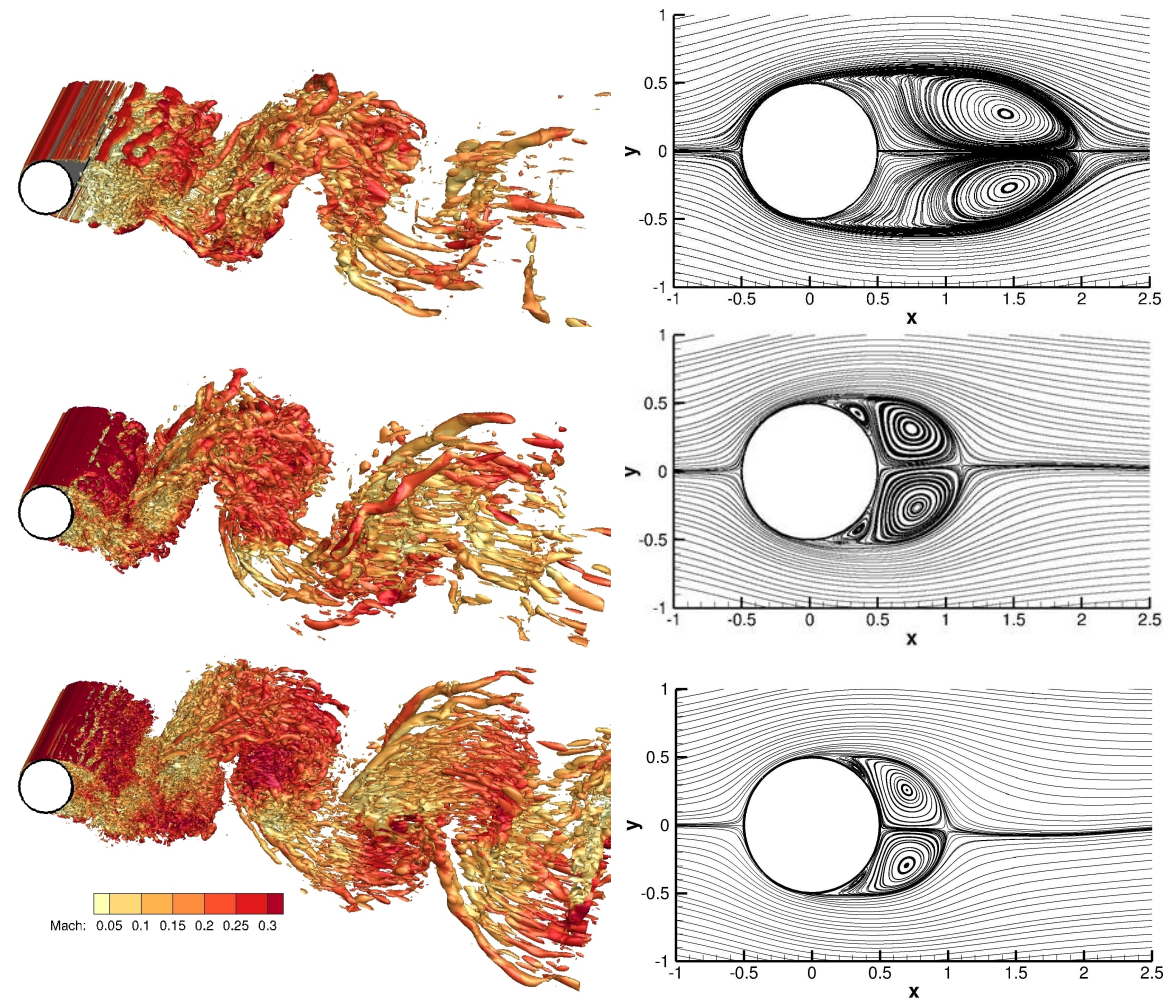

Figure 8: Flow past a circular cylinder at $R e=3900$ (top panel), $R e=20000$ (middle panel) and $R e=140000$ (bottom panel). Left: iso-surfaces of the Q-criterion coloured by the Mach number. Right: streamlines of mean flow field.

If we focus on the wall quantities, $C_{d}, C_{l, r m s}$, and $-C_{p, b}$, reported in Table 3 , we can observe an overall good agreement between all three DG simulations and the numerical and experimental reference data. In particular, the good match found for $C_{d}$ and $C_{l, r m s}$ in the case of the DG-WALE approach is consistent with the fact that the WALE model is able to provide the proper near-wall scaling [63]. As regards the DG-VMS simulation, these results also highlight the ability of the VMS approach to mitigate the well-known deficiency of the Smagorinsky model in the case of wall-bounded flows, namely, the spurious dissipation introduced by this model in laminar flow regions [68]. This property of the VMS method has already been pointed in [47]. In particular, the values of root-mean-square (rms) of the lift coefficient $C_{l, r m s}$ for the DG-VMS, as well as for the no-model simulations are in agreement with the uppermost value in the range provided by Norberg's experiment [64]. The fact that they are higher than the reference CFD values and that from the DG-WALE simulation might be interpreted as the result of a lower amount of numerical dissipation in these simulations. For all DG simulations, the values of the Strouhal number, $S_{t}$, and base pressure coefficient, $-C_{p, b}$, are in line with those of Lysenko et al. [51] and in agreement with the experimental 

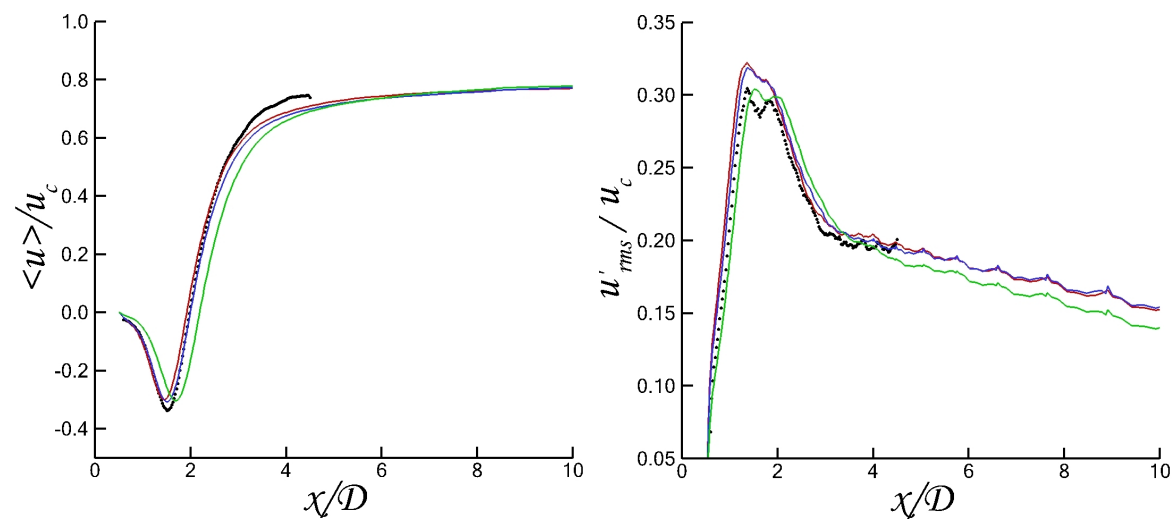

Figure 9: Cylinder flow at $R e=3900$ : velocity statistics on wake centreline. Left: mean streamwise velocity. Right: rms of streamwise velocity. • PIV, Parnaudeau et al. [67]; — no-model; — DG-VMS; DG-WALE.

values [67, 64].

As regards the length of the recirculation bubble, $L_{r}$, the values predicted by the DG simulations are within the range of those provided by the reference simulations. However, while the value obtained from the DG-VMS simulation $L_{r} / D=1.49$ is in excellent agreement with the experimental value reported in [67], the DG-WALE simulation yields a value of $L_{r} / D=1.69$ which is about $12 \%$ higher, closer to the value obtained by Lysenko et al. [51]. This overprediction of $L_{r}$ by the DG-WALE simulation is a consequence of the excessive dissipation introduced by this (mono-scale) model in the wake. This also explains the discrepancies found between the DG-WALE simulation and the experiment in the near-wake velocity statistics presented in the next section. Remark that this quantity is very sensitive to the amount of numerical and SGS dissipation in the computation. In fact, the no-model DG approach leads to the lowest value of $L_{r} / D=1.42$, while the use of the WALE model leads to the largest.

\subsubsection{First and second-order statistics}

To examine in more detail the performance of the different approaches considered, we have also compared the velocity statistics on the wake centreline, and at three stations in the recirculation region. These results are compared with the reference PIV data reported in [67]. The outcome from this comparison can be seen in Figs. 9 and 10.

We can see from the plot of mean streamwise velocity, in the left panel of Fig. 9, that the best match with the experimental data is obtained for the no-model and the DG-VMS simulations. The WALE model leads to a rightward shift of the velocity profile with respect to the experiment, leading to a longer recirculation bubble, as already pointed out in the previous section. The minimum of the axial velocity, $-U_{\min } / U_{c}$, takes a value of 0.30 for the DG-VMS and ILES simulations, and of 0.31 for the WALE simulation, in between the experiment [67] which yields 0.34 , and the value of 0.27 reported by Lysenko et al. [51]. The DG simulations therefore underpredict the experimental value by approximately $12 \%$. 
Table 4: Cylinder flow at $R e=20000$ : integral flow quantities.

\begin{tabular}{|c|c|c|c|c|c|c|c|c|c|c|}
\hline Method & Model & Mach & Mdofs & $\frac{\Delta r}{D(p+1)}$ & $T_{v s}$ & $C_{d}$ & $C_{l, r m s}$ & $-C_{p, b}$ & St & $\frac{L_{r}}{D}$ \\
\hline Exp & - & - & - & - & - & $1.14-1.20$ & $0.40-0.60$ & $1.07-1.20$ & 0.19 & - \\
\hline Wornom et al. [77] & VMS & 0.1 & 1.80 & $10^{-3}$ & 30 & 1.27 & 0.60 & 1.09 & 0.19 & 0.80 \\
\hline Lysenko et al. [52] & SGS & 0.2 & 12.40 & $5.610^{-4}$ & 75 & $1.33-1.36$ & $0.61-0.70$ & $1.11-1.14$ & $0.18-0.19$ & $0.57-0.69$ \\
\hline DG-VMS $O(5)$ & VMS & 0.2 & 2.59 & $10^{-2}$ & 95 & 1.39 & 0.64 & 1.42 & 0.19 & 0.67 \\
\hline
\end{tabular}

As regards the profiles of rms of the fluctuating streamwise velocity, $u_{r m s}^{\prime}$, the no-model and DG-VMS approaches are able to provide the correct position of the peak, although they overpredict its value by about $5 \%$. The standard LES, on the other hand, presents a peak value of $u_{r m s}^{\prime}$ close to the experimental value, although it fails to predict the correct position of the peak.

It is also obvious from these plots that, beyond approximately $x=3 D$, the turbulence levels are not well represented, which is a consequence of the large size of the elements in this region, as shown in Fig. 6 (see Sec. 6.1).

Figure 10 shows the mean and fluctuating velocity profiles at three stations in the near wake of the cylinder, namely, $x / D=1.06,1.54$ and 2.02. A quick look at these figures, and in particular at the plot of shear stresses, $\left\langle u^{\prime} v^{\prime}\right\rangle / U_{c}^{2}$, shows that the WALE simulation exhibits the most significant discrepancies with the experimental data. Even though the no-model simulation clearly outperforms the standard LES approach, it is the DG-VMS simulation that yields the best agreement with the experimental data. This is particularly visible form the profiles of Reynolds stresses.

\subsubsection{Energy spectra}

Finally, Fig. 11 compares the power spectra of the cross-stream velocity fluctuations from the three DG simulations with those reported by Parnaudeau et al. [67] at four locations on the wake centreline, namely, $x / D=3,5,7$, and 10 .

These plots reveal the presence of two well-marked peaks corresponding to two distinct frequencies, the fundamental frequency, located at $f / f_{s}=1$ and the second harmonic, located at $f / f_{s}=3$, in perfect agreement with the experiment. It is also worth noting that these two peaks prevail even in the further-wake regions. This is despite the clear loss of resolution as we move further down from the recirculation zone, as mentioned in the previous section. This is already noticeable at $x / D=5$, for which the energy content in the high-frequency range is clearly underestimated with respect to the experiment.

As expected, the larger amount of dissipation present in the standard LES computation is apparent from the lower energy levels exhibited at high wavenumbers, with respect to the two other simulations.

\subsection{Cylinder flow at $R e=20000$ : discussion of results}

Table 4 compares our DG-VMS results with those obtained by Lysenko et al. [52] using a second-order low-dissipative FV compressible solver at the same Mach number $M=0.2$ and for the same dimension of the computational domain as in the present simulation. For reference, the second-order VMS simulation results reported by Wornom et al. [77] are also shown in this table, as well as the experimental data reported 

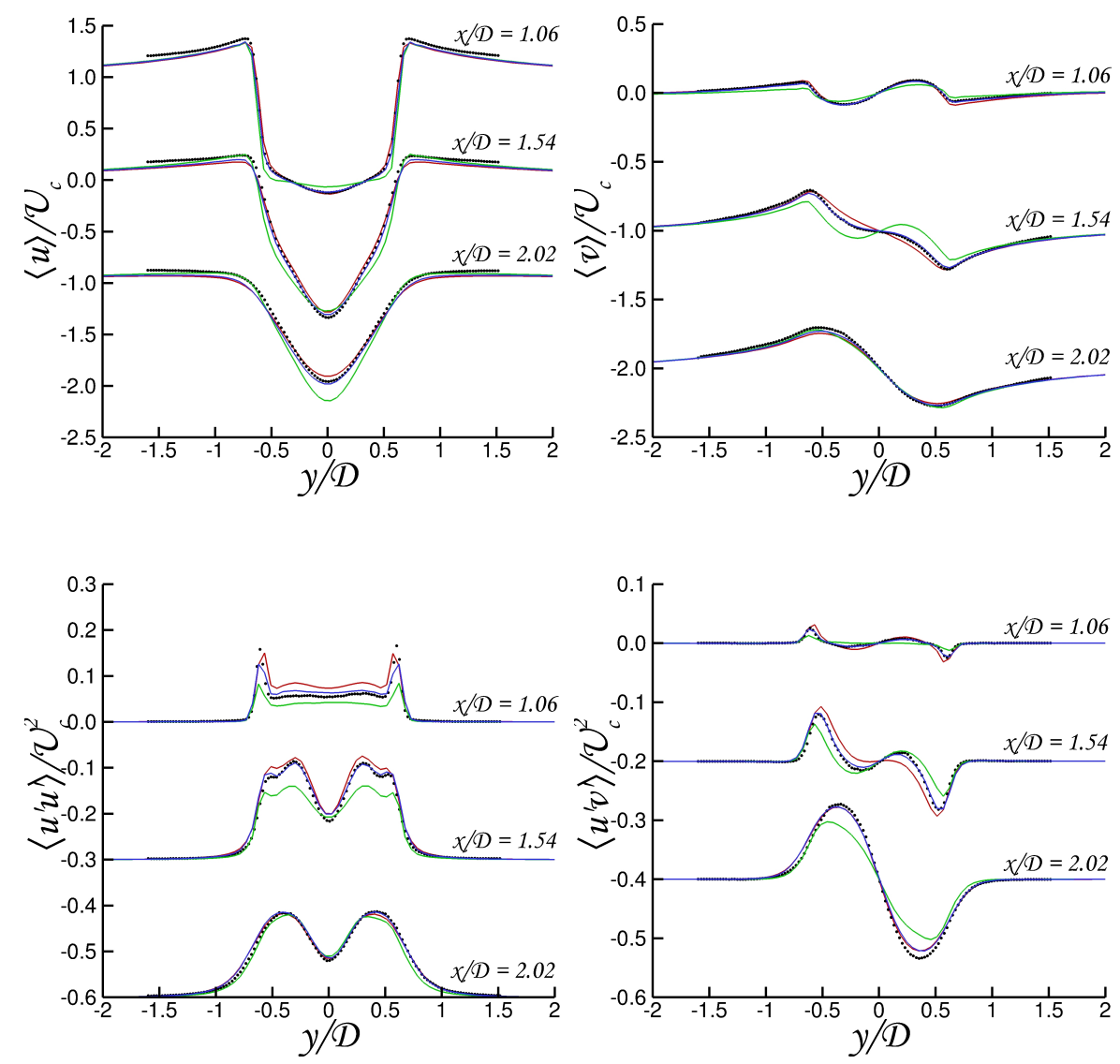

Figure 10: Cylinder flow at $R e=3$ 900: near-wake velocity statistics at $x / D=1.06,1.54$, and 2.02. $\bullet$ PIV, Parnaudeau et al. [67]; - no-model; — DG-VMS; - DG-WALE.

in $[76,48,64]$. The main integral flow quantities are compiled in this table. The range of values shown for the reference simulations correspond to the use of different numerical schemes and/or SGS modelling approaches. More details about these computations can be found in the original papers by the authors $[52,77]$.

We can see from this table that, overall, the results yielded by the DG-VMS fifthorder simulation are in fairly good agreement with the LES results by Lysenko et al. [52]. This is despite the fact that the DG simulation involves nearly five times fewer DOFs than the FV simulation. It is worth noting the much smaller effective size of the first grid cell at the wall, which in the DG simulation is about 18 times smaller with respect to the reference value. The good prediction achieved with our DG approach is partly a consequence of the excellent approximation properties of polynomial expansions in representing thin boundary layers, as pointed out by Gottlieb and Orszag [25].

This coarseness of the mesh in the vicinity of the wall does not seem to impact the accuracy with which the integral quantities, and in particular the length of the 

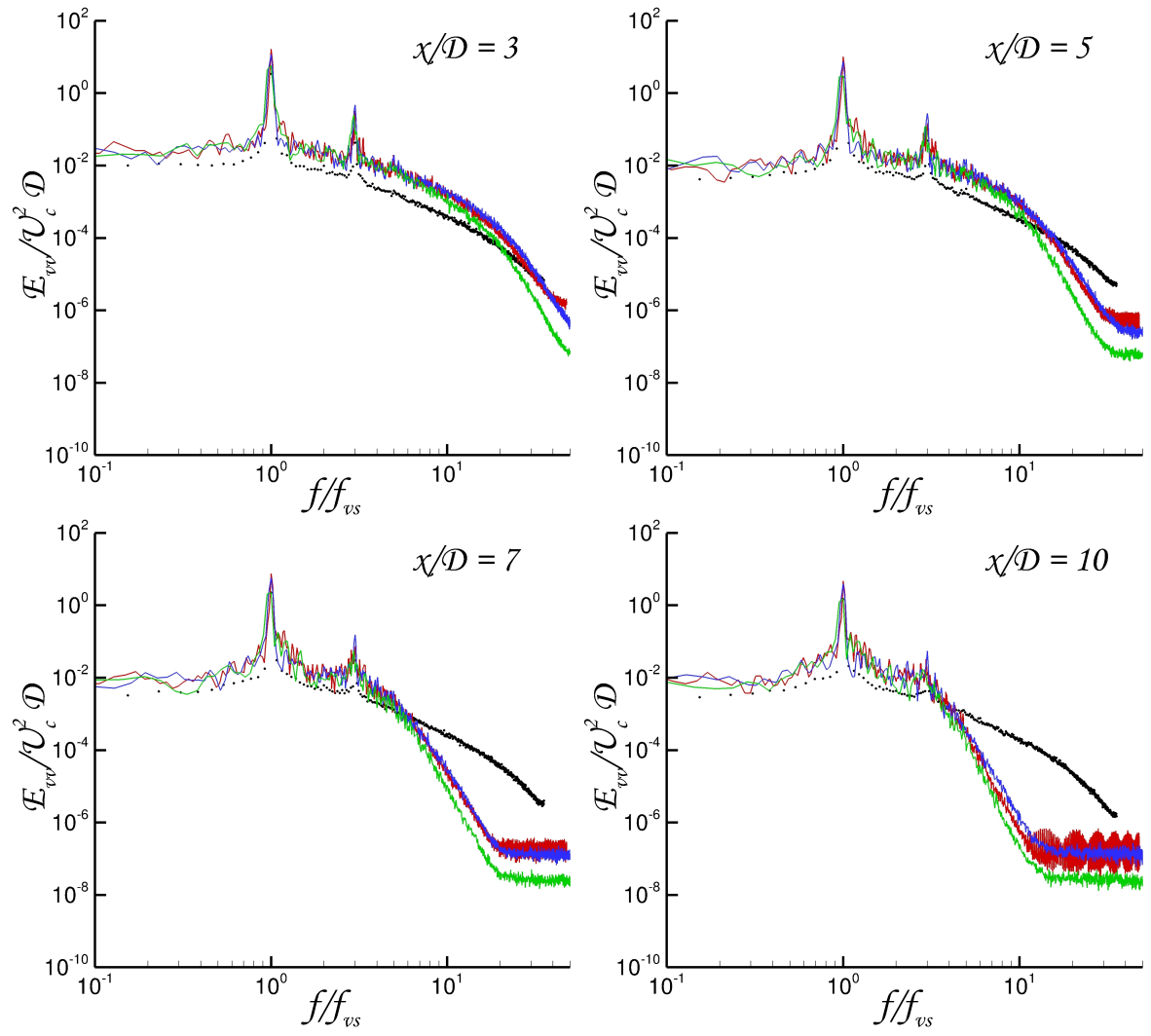

Figure 11: Cylinder flow at $R e=3900$ : energy spectra at $x / D=3,5,7$ and 10. - PIV, Parnaudeau et al. [67]; - no-model; - DG-VMS; - DG-WALE.

recirculation bubble $L_{r}$, are captured. In fact, the value of $L_{r}$ is well within the range of values reported by Lysenko et al. [52]. The length of the bubble obtained by Wornom et al. [77] is about $19 \%$ longer than in our simulation, which might be related to an insufficient averaging period [67, 45], or a larger amount of numerical dissipation in their simulation.

The main discrepancies with the CFD data are found for the value of the base pressure coefficient, $-C_{p, b}$, which in the DG simulation turns out to be $25 \%$ larger than the value reported in [52], and 18\% larger than the largest experimental value [64]. The important magnitude of this deviation is rather surprising, since this quantity is closely related to the drag coefficient, $C_{d}$, which appears in good agreement with the value reported by Lysenko et al. Indeed, the value of $C_{d}$ yielded by the DG simulation is only $2 \%$ above the upper-most value of the range reported in [52]. A good agreement with the reference data is also found for $C_{l, r m s}$, well within the range of values reported in [52], and only $6 \%$ above the upper range value in the experiment. The very different behaviour observed for these two integral quantities with respect to $-C_{p, b}$ can be justified 

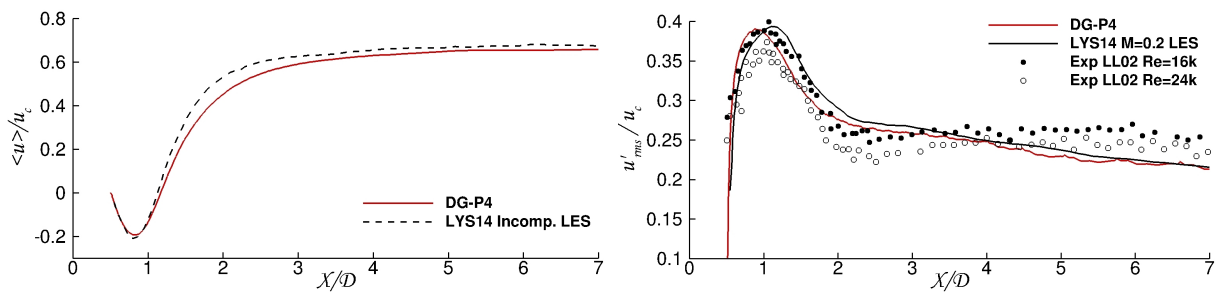

Figure 12: Cylinder flow at $R e=20000$ : streamwise velocity statistics on wake centreline. Left: mean streamwise velocity. Right: rms of streamwise velocity fluctuations. Red solid line: $D G-V M S$; black dashed line: incompressible FV simulation (TKE-I in [52]); black solid line: compressible FV simulation (SMAG- $\gamma$ in [52]); symbols: experimental data of Lim and Lee [48] at $R e=16000$ (dots) and $R e=24000$ (circles).

by noting that $C_{d}$ and $C_{l, r m s}$ are obtained by integration of the friction and pressure coefficients, respectively, over the cylinder wall. The base pressure coefficient, on the other hand, is a time-averaged point-value quantity, and thus much more sensitive to the under-resolution of the flow field in a localised region of the domain. The difference in the quality of the prediction of the integral quantities $C_{d}$ and $C_{l, r m s}$, with respect to $-C_{p, b}$ can be understood by the fact that the solution of the DG method converges in terms of the $L^{2}$-norm of the error (when the exact solution is smooth), and not in terms of the point-wise value of the error. The large discrepancy found for $-C_{p, b}$ might therefore be the consequence of a local lack of resolution in the wall-normal direction, in the cylinder-base region.

To further assess the quality of our results, the statistics in the near-wake region are also analysed. Figure 12 shows the mean streamwise velocity along the wake centreline (left), together with the profile of rms of the axial velocity fluctuations (right). The present results are compared with the available data from [52] and the experimental data of Son and Hanratty [74] for Reynolds numbers $R e=16000$ and 24000.

For the profile of mean streamwise velocity, the only CFD data available is that from an incompressible FV simulation using the dynamic $k$-equation SGS model (see [52] for more details). Despite the different physical models used, we can see from Fig. 12 that the two simulations are in relatively good agreement. As regards the profiles of rms of the fluctuating streamwise velocity, our results are compared with the compressible CFD results from [52] on a fine mesh and the experimental data of Son and Hanratty [74]. We can see that, overall, our results are in rather good agreement with the reference numerical data, and well within the range of the values predicted by the experiment. However, the departure from the experiment becomes apparent from approximately $x / D=2$, and is equally visible in the reference CFD data. This is partly a consequence of the lack of resolution in this region. It is interesting to note that this under-resolution does not seem to have a strong effect on the quality of the prediction in the wall region and near-wake dynamics.

\subsection{Static p-adaptive simulation of cylinder flow at $R e=140000$}

A DG-VMS simulation of the cylinder flow at $R e=140000$ and $M=0.2$ has been carried out based on a recently developed $p$-adaptation strategy [59]. The use 

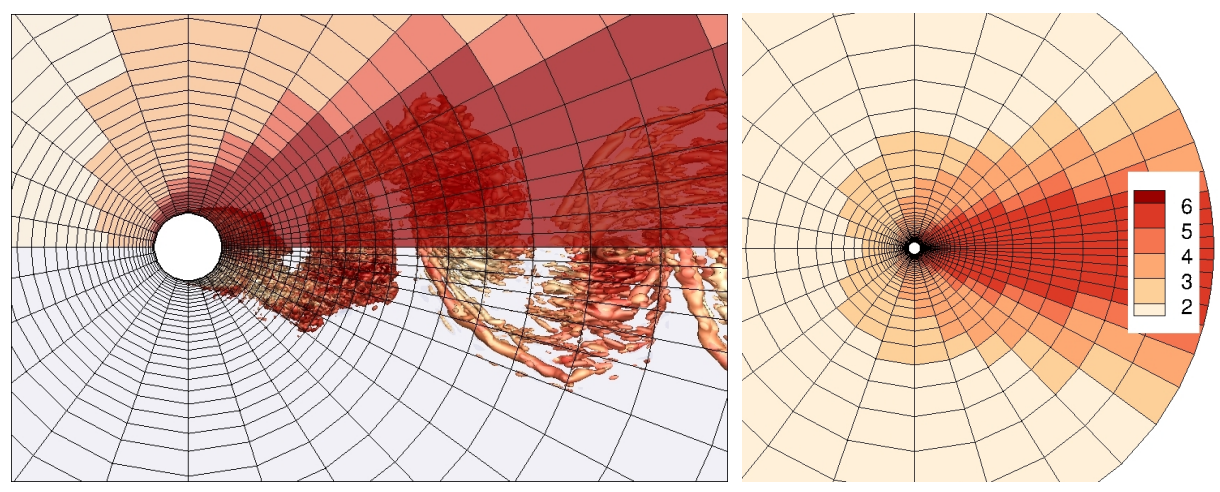

Figure 13: Flow past a cylinder at $\operatorname{Re}=140000$ : Distribution of polynomial degree $(p=2$ to 6$)$.

of $p$-adaptation, involving refinement as well as coarsening, allows for an important reduction of the number of DOFs with respect to uniform refinement, as well as an increased level of accuracy.

This higher Reynolds number corresponds to the upper subcritical regime for which the complexity of the flow increases considerably. In particular, the boundary layer becomes thinner, and the separated shear layer transitions shortly after separation generating a stronger fully turbulent vortex shedding.

In the next sections a brief description of the p-adaptive algorithm implemented in the Aghora solver is provided, followed by the main results from the DG-VMS simulation performed at this higher Reynolds number.

\subsubsection{Description of the p-adaptation algorithm}

The $p$-adaptive algorithm employed here is similar to that developed in [59]. The error estimator used in this work is the small-scale energy density (SSED) estimator proposed in [59], which can also be interpreted as a measure of the kinetic energy associated with the highest-order modes representing the solution within the element.

The algorithm is initialised by specifying a uniform polynomial degree distribution. A first simulation is then carried out based on the prescribed polynomial degree that will serve as initial solution for the next iteration of the $p$-adaptive algorithm. At each iteration of the refinement algorithm the simulation is let to evolve until the statistically steady state is reached. The SSED indicator is then evaluated from the instantaneous solution over a sufficiently long integration time and its $L^{\infty}$-norm over time is used as refinement indicator. An element $K$ is then marked for refinement if the value of the refinement indicator $\eta_{K}>\eta_{\text {ref }}$ where $\eta_{\text {ref }}$ denotes the volume-weighted average of the refinement indicator over the domain. Coarsening is also allowed by introducing a local coarsening indicator $\xi_{K}$. The coarsening indicator is here defined similarly to the SSED indicator as the $L^{2}$-norm of the energy contained in modes corresponding to both the $(p-1)^{t h}$ and $p^{t h}$ order of the instantaneous solution normalised by the square root of the cell volume. An element is then marked for coarsening if $\xi_{K}<\varepsilon$, where $\varepsilon$ is a user-defined threshold. This procedure leads to the selection for refinement of elements with relatively high values of the error while enforcing a conservative 
criterion for coarsening. In the simulation presented in the next section, the refinement and coarsening thresholds are, respectively, $\eta_{\text {ref }}=1.15 \cdot 10^{-3}$ and $\varepsilon=2 \cdot 10^{-4}$, at the second iteration of the refinement algorithm.

For the simulations presented in this section, an initial uniform distribution of polynomial degree $p=4$ is used. Two iterations of the adaptive algorithm have been performed leading to the $p$-pattern shown in Fig. 13, with $p$ varying from 2 to 6 . The adaptive algorithm allows for the automatic coarsening of the resolution in the laminar region ahead of the cylinder and refinement in the turbulent region fully enveloping the vortex shedding structures (as seen in the left panel of Fig. 13).

In order to simplify the averaging procedure, the polynomial degree $p$ is kept constant in the spanwise direction. This leads to a total number of 4.98 million DOFs. We remark that if we were using uniform $p$ refinement on the same grid, the necessary number of DOFs to attain a similar level of accuracy at $p=6$ would be of 7.11 million, i.e. $42 \%$ higher. As seen from Fig. 13, the polynomial degree in the first element layer around the cylinder is $p=6$. This yields an effective size of the first element at the wall of $\Delta r /(p+1)=4 \cdot 10^{-3} D$.

As regards the order of the quadrature rule, the local number of quadrature points is adapted depending on the local polynomial degree and corresponds to $q=p+1+m$ with $m=\{2,2,3,4,4\}$ for $p=2$ to 6 .

In order to achieve high parallel efficiency throughout the simulation, the computational grid has been partitioned taking into account the uneven distribution across the domain of the polynomial degree $p$ and of the number of quadrature points $q$. The weights used in the graph-partitioning problem are derived from direct measurements of the performance of the employed solver in a simplified configuration. This simplified problem is based on the same numerical parameters ( $p, q$, numerical flux, etc.) and the same physical model as the present simulation. Following this approach, the $p$-adapted computational $h p$-grid is split into 3360 domains, leading to a parallel efficiency of $87 \%$. The reader is referred to [60] for more details on the specific mesh partitioning strategy employed in this work.

\subsubsection{Cylinder flow at $R e=140$ 000: discussion of results}

The $p$-adapted DG solution is compared with the numerical data produced by Breuer [10] and Fröhlich et al. [24] using the incompressible second-order FV solver LESOCC [8], as well as the experimental data from Cantwell and Coles [12]. In [10], the author performed a number of simulations using different levels of resolution and spanwise extensions of the computational domain. Here we consider for comparison the simulations $C 3$ and $D 3$ corresponding to a spanwise length of the cylindrical domain of $\pi D$ and a radial extension of $15 D$. The number of DOFs was 1.74 and $6.76 \mathrm{Mdofs}$, respectively, for simulations $C 3$ and $D 3$. They both employ the Smagorinsky SGS model. As regards the numerical data obtained by Fröhlich et al., the LES simulation denoted RUN2 in [24] is also considered. This simulation is based on the Smagorinsky model and employs 2.19 Mdofs in a computational domain with a spanwise extension of $L_{z}=4 D$.

Table 5 compiles the values of the integral quantities obtained from the present simulation and for the reference data used for comparison. As regards the values of 
Table 5: Circular cylinder flow at $\mathrm{Re}=140000$ : comparison of integral flow quantities with the simulation results of Breuer [10] and Fröhlich et al. [24], and the experimental data of Cantwell and Coles [12].

\begin{tabular}{lccrrrrrc}
\hline Data & $L_{z} / D$ & Mdofs & $\frac{\Delta r}{D(p+1)}$ & $N_{v s}$ & $C_{d}$ & $-C_{p, b}$ & $S t$ & $\frac{L_{r}}{D}$ \\
\hline Experiment [12] & - & - & - & - & 1.24 & 1.21 & 0.18 & 0.50 \\
Fröhlich-R2[24] & 4 & 2.19 & $1.710^{-4}$ & $10-14$ & 1.25 & 1.40 & 0.21 & 0.59 \\
Breuer-C3[10] & $\pi$ & 1.74 & $410^{-4}$ & $13-34$ & 1.28 & 1.51 & 0.22 & 0.46 \\
Breuer-D3[10] & $\pi$ & 6.76 & $410^{-4}$ & $13-34$ & 1.37 & 1.60 & 0.21 & 0.42 \\
DG-VMS $O(3-7)$ & $\pi$ & 4.98 & $410^{-3}$ & 71 & 1.43 & 1.59 & 0.19 & 0.50 \\
\hline
\end{tabular}

drag, $C_{d}$, and base pressure, $-C_{p, b}$, coefficients, our results are in good agreement with the CFD data from Breuer, though substantially larger than the experimental values and those reported in [24]. The Strouhal number, $S t$, is in between the experimental and the numerical reference values. The $S t$ measured by Cantwell and Coles [12] appears, however, very low with respect to what has been found in other experimental works. In particular, Son and Hanratty [74] report a value slightly above 0.19 which is consistent with the value found in the present work. Finally, the recirculation length, $L_{r}$, predicted by the DG simulation is in perfect agreement with the experimental value and within the range of the reference numerical values. We have however observed, that the value of this quantity is very sensitive to the averaging period, in agreement with the observations made in [67]. This might explain the differences found between our value and those reported in [10] and [24], who considered an averaging time considerably lower. As pointed out in [67], and from our own observations, this quantity is actually a very reliable indicator of the level of accuracy in the simulation. It is indeed very sensitive to the mesh resolution in the near-wake region, as well as to the amount of numerical and SGS dissipation in the simulation.

As regards the rms of the drag and lift coefficients we obtain the following values, $C_{d}^{\prime}=0.094$ and $C_{l}^{\prime}=0.64$. For this particular Reynolds no values for these quantities have been reported in the literature.

In Fig. 14, the wake centreline statistics are also compared with those obtained from the experiment. As regards the mean velocity profile, we can see that the length of the recirculation region matches very well the experimental value (see also Table 5). It is apparent from these results, however, that as we move further downstream from the recirculation region, the streamwise velocity levels are significantly under-predicted. This is partly due to the very coarse mesh used in the wake beyond the recirculation region. This is in contrast with the profile of the streamwise Reynolds stress $\left\langle u^{\prime} u^{\prime}\right\rangle$, better predicted over a longer distance beyond the recirculation zone. This might be a consequence of the higher levels, with respect to the reference, found in the region closer to the cylinder base. As regards the cross-stream Reynolds stress $\left\langle v^{\prime} v^{\prime}\right\rangle$, their magnitude is significantly higher than the experimental value, although in fair agreement with the values predicted by Breuer on his fine-grid simulations (see [10], not shown here). The overshoot of $\left\langle v^{\prime} v^{\prime}\right\rangle$ observed in the vicinity of the cylinder base is also present in Breuer's results and in the simulations reported in [24]. No physical argument can be provided to explain this behaviour, which we suspect to be a numerical artifact.

The wake statistics at a location $x=1$ have also been inspected and compared to 

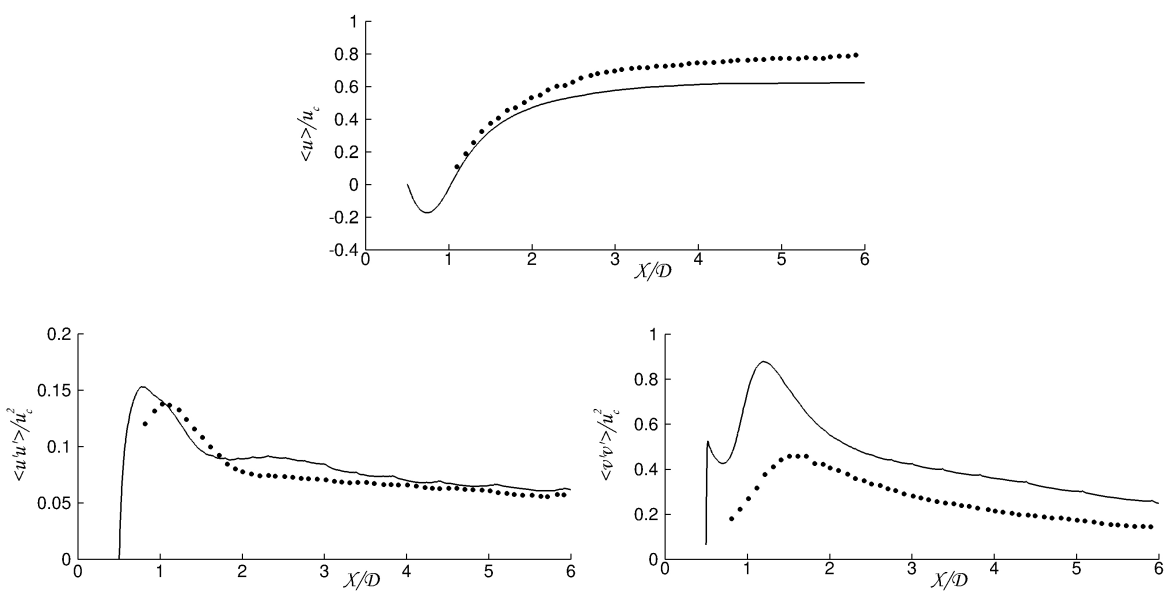

Figure 14: Flow past a circular cylinder at $\mathrm{Re}=140000$. Wake centreline statistics. $\bullet$ : Experiments [12], solid line: $p$-adapted DG-VMS.
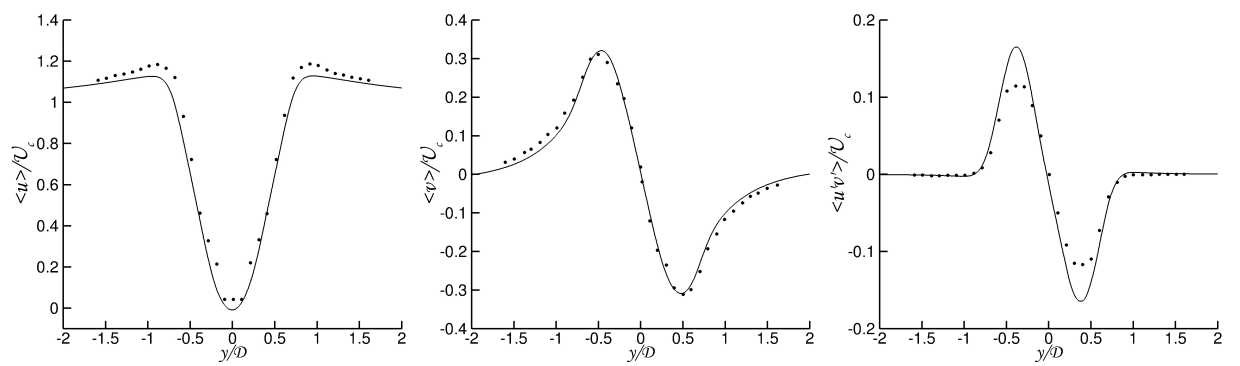

Figure 15: Flow past a circular cylinder at $\operatorname{Re}=140000$. Wake statistics at $x=1 . \bullet:$ Experiments [12], solid line: $p$-adapted DG-VMS.

the experimental data of Cantwell and Coles [12]. The outcome from this comparison can be seen in Fig. 15. We can observe the overall good agreement found with the experiment for these quantities. The most significant discrepancies appear in the profile of the shear stresses $\left\langle u^{\prime} v^{\prime}\right\rangle$, although their magnitude is in line with the differences found by Breuer on his fine-grid simulations. These discrepancies might be linked to the different conditions between the experiment and the simulations (e.g. zero turbulence level at the inlet in the simulation). In fact, regarding the study carried out in [10], the author mentioned that grid refinement did not appear to provide convergence towards the experimental values.

\section{Conclusions}

The scale-resolving capabilities of a high-order multiscale DG method based on the VMS approach to LES $[14,20]$ have been assessed through simulations of the flow past a circular cylinder in the sub-critical regime at $R e=3900,20000$, and 140000 . 
The effect of the numerical flux function on the accuracy of the DG-LES solutions has been investigated by performing ILES and VMS simulations of the TGV configuration at $R e=20000$ considering different Riemann solvers. It appears from this research that the use of the DG-VMS approach in combination with the least dissipative possible numerical flux leads to the best match with the reference data, with a slight advantage for the low-dissipative Roe scheme over the LLF scheme. We have also put forward the strong influence of the numerical flux function on the no-model DG approach for under-resolved simulations when the Reynolds number is high, and the much more robust behaviour of the VMS approach. Finally, we have highlighted the importance of examining each of the terms involved in the balance equation, rather than focusing on the analysis of an isolated quantity such as the k.e. dissipation, or even the LS dissipation (or equivalently the enstrophy) alone.

The outcome of the cylinder flow DG-VMS computations at the three Reynolds numbers considered show an overall good agreement with the reference high-resolution LES data, in terms of integral flow quantities and near-wake statistics. This is despite the fact that the DG-VMS simulations involve much fewer DOFs than the reference LES. We have also highlighted, for the lowest Reynolds number, $R e=3900$, the over-dissipative character of the standard DG-LES as compared to DG-VMS approach.

It is noteworthy that the effective height of the first grid cell off the wall in the DG simulations is one order of magnitude larger than in the reference LES. This highlights the excellent approximation properties of DG approximations in representing boundary layers on coarse grids.

The promising results obtained form the $p$-adapted DG-VMS simulation of the cylinder flow at $R e=140000$ demonstrate the great potential of $p$-adaptive DG methods for the simulation of complex unsteady phenomena. This is an encouraging step towards gradually making high-order DG methods available to the aerospace industry.

\section{Acknowledgments}

This work was partly funded through the TILDA (Towards Industrial LES/DNS in Aeronautics - Paving the Way for Future Accurate CFD) project grant agreement number 635962, receiving funding from the European Union's Horizon 2020 research and innovation program. This work was granted access to the HPC resources of TGCC/CINES under the allocations A0022A10129, A0032A10309 and A0012A07624/A0032A07624 made by GENCI.

\section{References}

[1] Abbà, A., Bonaventura, L., Nini, M. and M. Restelli (2015) Dynamic models for Large Eddy Simulation of compressible flows with a high-order DG method, Comput Fluids 122:209-222.

[2] Ahmed N., Chacón Rebollo T., John V. and Rubino S. (2017) A review of variational multiscale methods for the simulation of turbulent incompressible flows, Arch Comput Meth Eng 24:115-164. 
[3] Arnold D. N., Brezzi F., Cockburn B. and Marini L. D. (2001) Unified Analysis of Discontinuous Galerkin Methods for Elliptic Problems, SIAM J Numer Anal 39:1749-1779.

[4] Bando K, Naddei F., de la Llave Plata M. and Ihme M. Variational multiscale SGS modeling for LES using a high-order DG Method, Annual Research Briefs, Center for Turbulence Research, Stanford, 2018.

[5] Bassi F. and Rebay S. (1997) A high-order accurate discontinuous finite element method for the numerical solution of the compressible Navier-Stokes equations, $\mathrm{J}$ Comput Phys 131(2): 267-279.

[6] Bassi F., Botti L., Colombo A., Di Pietro D. A. and Tesini P. (2012) On the flexibility of agglomeration based physical space DG discretizations, J Comput Phys 231(1):45-65.

[7] Beck A., Flad D., Tonhäuser C., Gassner G. and Munz C.-D. (2016) On the influence of polynomial de-aliasing on subgrid scale models, Flow Turbul Combust 97(2):475-511.

[8] Breuer M. and Rodi W. (1994) Large-eddy simulation of turbulent flow through a straight square duct and a $180^{\circ}$ bend. In: Voke P. R., Kleiser L., Chollet J. P. (eds) Direct and Large-Eddy Simulation I. Fluid Mechanics and Its Applications, vol 26. Springer, Dordrecht

[9] Breuer M. (1998) Large-eddy simulation of the subcritical flow past a circular cylinder: numerical and modeling aspects, Int J Numer Meth Fluids 28(9):02712091.

[10] Breuer M. (2000) A challenging test case for large-eddy-simulation: high Reynolds number circular cylinder flow, Int J Heat Fluid Flow 21(5):648-654.

[11] Brezzi F., Cockburn B., Marini L. D. and Süli E. (2006) Stabilization mechanisms in discontinuous Galerkin finite element methods, Comput Meth Appl Mech Eng 195(25-28): 3293-3310.

[12] Cantwell B. and Coles D. (1983) An experimental study of entrainment and transport in the turbulent near wake of a circular cylinder, J Fluid Mech 136:321374.

[13] Chapelier J.-B., Développement et évaluation de la méthode de Galerkin discontinue pour la simulation des grandes échelles des écoulements turbulents, PhD Thesis, Université de Bordeaux, 2013.

[14] Chapelier J.B., de la Llave Plata M., Renac F. and Lamballais E. (2014) Evaluation of a high-order discontinuous Galerkin method for the DNS of turbulent flows, Comput Fluids 95:210-226.

[15] Chapelier J.-B., de la Llave Plata M. and Lamballais E. (2016) Development of a multiscale LES model in the context of a modal DG method, Comput Meth Appl Mech Eng 307:275-299. 
[16] Cockburn B. (2003) Discontinuous Galerkin methods, J Appl Math Mech 83(11):731-754.

[17] Collis S. S. (2001) Monitoring unresolved scales in multiscale turbulence modeling, Phys Fluids 13:1800-1806.

[18] Collis S.S. (2002) The DG/VMS method for unified turbulence simulation, AIAA Paper 2002-3124.

[19] Dairay T., Lamballais E., Laizet S. and Vassilicos J. C. (2017) Numerical dissipation vs. subgrid-scale modelling for LES, J Comput Phys 337:252-274.

[20] de la Llave Plata M., Couaillier V. and Le Pape M.-C. (2018) On the use of a high-order discontinuous Galerkin method for DNS and LES of wall-bounded turbulence, Comput Fluids 176:320-337.

[21] Ducros F., Comte P. and Lesieur M. (1996) Large-eddy simulation of transition to turbulence in a boundary layer developing spatially over a flat plate, J Fluid Mech 326:1-36.

[22] Ferrer E. (2017) An interior penalty stabilised incompressible discontinuous Galerkin-Fourier solver for implicit large eddy simulations, J Comput Phys 348:754-775.

[23] Farhat C., Rajasekharan A. and Koobus B. (2006) A dynamic variational multiscale method for large eddy simulations on unstructured meshes, Comput Meth Appl Mech Eng 195(13-16):1667-1691.

[24] Fröhlich J., Rodi W., Bertoglio J. P., Bieder U. and Touil H. (2001) LES of flow around circular cylinders on structured and unstructured grids, II. In: Hirschel E. H. (eds) Numerical Flow Simulation II. Notes Numer Fluid Mech 75, Springer.

[25] Gottlieb D. and Orszag S. A., Numerical analysis of spectral methods : theory and applications, SIAM, 1977.

[26] Gravemeier V., Wall W. A. and Ramm E. (2004) A three-level finite element method for the instationary incompressible Navier-Stokes equations, Comput Meth Appl Mech Eng 193(15-16):1323-1366.

[27] Gravemeier V. (2007) Variational multiscale large-eddy simulation of turbulent flow in a diffuser, Comput Mech 39(4):477-495.

[28] Hartmann R. and Houston P. (2008) An optimal order interior penalty DG discretization of the compressible Navier-Stokes equations, J Comput Phys 227(22):9670-9685.

[29] Hillewaert K., Development of the discontinuous Galerkin method for highresolution, large scale CFD and acoustics in industrial geometries, PhD thesis, Université Catholique de Louvin, 2013. 
[30] Holmen J., Hughes T. J. R., Oberai A. A. and Wells G. N. (2004) Sensitivity of the scale partition for variational multiscale large-eddy simulation of channel flow, Phys Fluids 16(3):824-827.

[31] Hughes T. J. R., Feijóo G. R., Mazzei L. and Quincy J. B.(1998) The variational multiscale method - a paradigm for computational mechanics, Comput Meth Appl Mech Eng 166(1):3-24.

[32] Hughes T. J. R., Mazzei L. and Jansen K. E. (2000) Large-eddy simulation and the variational multiscale method, Comput Visual Sci 3(1-2):47-59.

[33] Hughes T. J. R., Mazzei L., Oberai A. A. and Wray A. A. (2001) The multiscale formulation of large-eddy simulation: Decay of homogeneous isotropic turbulence, Phys Fluids 12(2):505-512.

[34] Hughes T. J. R., Oberai A. A. and Mazzei L. (2001) Large-eddy simulation of turbulent channel flows by the variational multiscale method, Phys Fluids 13(6):1784-1799.

[35] Hulshoff S. J., Munts E. A. and Labrujere J. (2011) Wall-stress boundary conditions for variational-multiscale LES, Int J Numer Meth Fluids, 66:1341-1353.

[36] Huynh H. T. (2007) A flux reconstruction approach to high-order schemes including discontinuous Galerkin methods. AIAA Paper 2007-4079.

[37] Huynh H. T. (2009) A reconstruction approach to high-order schemes including discontinuous Galerkin for diffusion. AIAA Paper 2009-403.

[38] Itam E., Wornom S., Koobus B. and Dervieux A. (2019) Hybrid versus pure-LES models comparison for subcritical cylinder flows. In: M.-V. Salvetti et al. (eds.) Direct and Large-Eddy Simulation XI, ERCOFTAC Series, Springer.

[39] Jansen K. and Tejada-Martínez A. (2002) An evaluation of the multiscale model for large-eddy simulation using a hierarchical basis, AIAA Paper 2002-0283.

[40] John V. and Kaya S. (2005) A finite-element variational multiscale method for the Navier-Stokes equations. SIAM J Sci Comp 26:1485-1503.

[41] Karabelas S. J., LES of high-Reynolds number flow past a rotating cylinder (2010) Int J Heat Fluid Flow 31(4):518-527.

[42] Koobus B. and Farhat C. (2004) A variational multiscale method for the large-eddy simulation of compressible turbulent flows on unstructured meshes - application to vortex shedding, Comput Meth Appl Mech Eng 193(15-16):1367-1383.

[43] Levasseur V., Sagaut P., Chalot F. and Davroux A. (2006) An entropy-variablebased VMS/GLS method for the simulation of compressible flows on unstructured grids, Comput Meth Appl Mech Eng 195(9-12):1154-1179. 
[44] Lamballais E., Dairay T., Laizet S. and Vassilicos J. C. (2019) Implicit/explicit spectral viscosity and large-scale SGS effects. In: M.-V. Salvetti et al. (eds) Direct and Large-Eddy Simulation XI, ERCOFTAC Series, Springer.

[45] Lehmkuhl O., Rodríguez I., Borrell R. and Oliva A. (2013) Low-frequency unsteadiness in the vortex formation region of a circular cylinder, Phys Fluids 25(8):085109.

[46] Lesieur M., Métais O. and Comte P., Large-eddy simulation of turbulence, Cambridge University Press, Cambridge 2005.

[47] Lévêque E., Toschi F., Shao L. and Bertoglio J. (2007) Shear-improved Smagorinsky model for large-eddy simulation of wall-bounded turbulent flows, J Fluid Mech 570:491-502.

[48] Lim H. C. and Lee S. J. (2002) Flow control of circular cylinders with longitudinal grooved surfaces, AIAA J. 40(10):2027-2036.

[49] Lodato G. and Chapelier J.-B. (2018) Evaluation of the Spectral Element Dynamic Model for Large-Eddy Simulation on Unstructured, Deformed Meshes, Flow Turbul Combus 101(2):271-294.

[50] Lv Y. and Ihme M. (2015) Entropy-bounded discontinuous Galerkin scheme for Euler equations, J Comput Phys 295:715-739.

[51] Lysenko D. A., Ertesvåg I. S. and Rian K. E. (2012) LES of the flow over a circular cylinder at Reynolds number 3900 using the OpenFOAM toolbox. Flow Turbul Combust 89(4):491-518

[52] Lysenko, D. A., Ertesvåg, I. S. and Rian, K. E. (2014) LES of the flow over a circular cylinder at Reynolds number $2 \times 10^{4}$, Flow Turbul Combust 92(3):673698.

[53] Mengaldo G., Moura R. C., Giralda B., Peiró J. and Sherwin S. J. (2018) Spatial eigensolution analysis of discontinuous Galerkin schemes with practical insights for under-resolved computations and implicit LES, Comput Fluids 169:349-364.

[54] Moura R. C., Peiró J. and Sherwin S. J. (2015) Modified equation analysis for the discontinuous Galerkin formulation. In: R. M. Kirby, M. Berzins, J. S. Hesthaven (eds) Spectral and High Order Methods for Partial Differential Equations, ICOSAHOM 2014, Springer, pp. 375-383.

[55] Moura R. C., Mengaldo G., Peiró J. and Sherwin S. J. (2017) On the eddyresolving capability of high-order discontinuous Galerkin approaches to implicit LES / under-resolved DNS of Euler turbulence, J Comput Phys 330:615-623.

[56] Moussaed C., Wornom S., Salvetti M.-V., Koobus B. and Dervieux A. (2014) Impact of dynamic subgrid-scale modeling in variational multiscale large-eddy simulation of bluff-body flows, Acta Mech 225:3309. 
[57] Munts E. A., Hulshoff S. J. and de Borst R. (2007) A modal-based multiscale method for large eddy simulation, J Comput Phys 224(1):389-402.

[58] Naddei F., de la Llave Plata M., Lamballais E., Couaillier V., Massot M. and Ihme M., Large-scale space definition for the DG-VMS method based on energy transfer analyses. In Proc. of the Summer Program, Center for Turbulence Research, Stanford University, 2018.

[59] Naddei F., de la Llave Plata M. and Couaillier V. (2019) A comparison of refinement indicators for p-adaptive simulations of steady and unsteady flows using DG methods, J Comp Phys 376:508-533.

[60] Naddei F., Adaptive large-eddy simulations based on discontinuous Galerkin methods, PhD thesis, École Polytechnique, October 2019.

[61] Naddei F., de la Llave Plata M. and Lamballais E., Spectral and modal energy transfer analyses of the discontinuous Galerkin Variational Multiscale approach. Under review in J Comput Physics.

[62] Navah F., de la Llave Plata M. and Couaillier V., A high-order variational multiscale approach to turbulence for compact nodal schemes. Under review in Comput Meth Appl Mech Eng. arXiv preprint arXiv:1809.03966v1.

[63] Nicoud F. and Ducros F. (1999) Subgrid-Scale Stress Modelling Based on the Square of the Velocity Gradient Tensor, Flow Turbul Combust 62:183-200.

[64] Norberg C. (2003) Fluctuating lift on a circular cylinder: review and new measurements, J Fluids Struct 17:57-96.

[65] Oberai A. and Hughes T. J. R. (2002) The variational multiscale formulation of LES: channel flow at $\operatorname{Re}=590$, AIAA Paper 2002-1056.

[66] Ouvrard H., Koobus B., Dervieux A. and Salvetti M.-V. (2010) Classical and variational multiscale LES of the flow around a circular cylinder on unstructured grids, Comput Fluids 39:1083-1094.

[67] Parnaudeau P., Carlier J., Heitz D. and Lamballais E. (2008) Experimental and numerical studies of the flow over a circular cylinder at Reynolds number 3900, Phys Fluids 20(8):085101.

[68] Piomelli U. and Zang T. A. (1991) Large-eddy simulation of transitional channel flow, Comput Phys Commun 65:224-230.

[69] Rasthofer U. and Gravemeier V. (2018) Recent Developments in Variational Multiscale Methods for Large-Eddy Simulation of Turbulent Flow, Arch Comput Meth Eng, 25(3):647-690.

[70] Roe P. L. (1981) Approximate Riemann solvers, parameter vectors, and difference schemes, J Comput Phys 43(2):357-372. 
[71] Rusanov V. V. (1961) Calculation of interaction of nonsteady-shock waves with obstacles, J Comput Math Phys, USSR, 1:267-279.

[72] Salvatici E. and Salvetti M.-V. (2003) Large-eddy simulations of the flow around a circular cylinder: effects of grid resolution and subgrid scale modeling, Wind and Structures, 6(6):419-436.

[73] Smagorinsky J. (1963) General Circulation Experiments with the Primitive Equations, Mon Weather Rev 91(3):99-164.

[74] Son J. and Hanratty T. (1969) Velocity gradients at the wall for flow around a cylinder at Reynolds numbers from $5 \times 10^{3}$ to $10^{5}$, J Fluid Mech 35:353-368.

[75] Wasberg C. E., Gjesdal, T., Reif, B. A. P. and Andreassen, O. (2009) Variational multiscale turbulence modelling in a high order spectral element method, J Comput Phys 228(19):7333-7356.

[76] West G. S. and Apelt C. J. (1993) Measurements of Fluctuating Pressures and Forces on a circular cylinder in the Reynolds Number Range $10^{4}$ to $2 \cdot 5 \times 10^{5}, \mathrm{~J}$ Fluids Struct 7(3):227-244.

[77] Wornom S., Ouvrard H., Salvetti M.-V., Koobus B. and Dervieux A. (2011) Variational multiscale LES of the flow past a circular cylinder: Reynolds number effects, Comput Fluids 47(1):44-50.

[78] Yser P., Barré S., Dagrau F., Chalot F. and Bailly C. (2016) High-order Variational Multiscale Model with an Explicit Filtering in a Stabilised Finite Element Method for LES/DES Computations, AIAA Paper 2016-2722.

[79] Yser P. and Bailly C. (2018) High-order variational multiscale model in finite elements applied to the LEISA-2 configuration, AIAA J 56(12):5000-5012. 